\title{
Ergebnisse einer Online-Umfrage zur „Angemessenheit von Aussprachevarianten in formellen Sprechsituationen“
}

\author{
Stefan Kleiner
}

\begin{abstract}
Im Beitrag werden die Ergebnisse einer im Jahr 2015 durchgeführten OnlineUmfrage vorgestellt, in der die Angemessenheit von Aussprachevarianten des Deutschen in formellen Sprechsituationen bewertet werden sollte. $\mathrm{Zu}$ diesem Zweck wurden den 1.964 Teilnehmer/-innen Aussprachevarianten von insgesamt 207 Lexemen vorgelegt, vor allem aus den Bereichen Wortakzent, Vokalquantität und Fremdwortrealisierung. Die Umfrageergebnisse werden tabellarisch aufgeführt und damit weiterer Forschung zur Verfügung gestellt.
\end{abstract}

Keywords Deutsche Aussprache, Variation, Hochdeutsch, Standardsprache, Deutscher Sprachraum, Online-Umfrage, Duden-Aussprachewörterbuch

\section{Inhalt}

1. Überblick 2

2. Aufbau und Inhalt der Umfrage

3. Zum Problem der regionalen Herkunft der Umfrageteilnehmer/-innen 5

4. Die Faktoren Geschlecht, Alter, Schulbildung und Beruf 8

5. Die Umfrageergebnisse 9

5.1 Teil 1: Betonte Silbe

5.2 Teil 2: Vokalquantität _ 22

5.3 Teil 3: Gemischte Phänomene __ 34

5.4 Teil 4: Lehnwörter 47

6. Schluss 69

Literatur 69

Bibliografische Informationen $\quad 70$

Autorendaten 70

Impressum $\quad 70$ 


\section{1. Ůberblick}

Im Frühsommer 2015 wurde im Rahmen der Neubearbeitung des Duden-Aussprachewörterbuchs (Kleiner/Knöbl/Mangold 2015) vom damaligen Projektteam eine Online-Umfrage ins Netz gestellt, in der dazu aufgerufen wurde, Aussprachevarianten aus verschiedenen lautlichen Domänen hinsichtlich ihrer Angemessenheit in formellen Sprechsituationen zu beurteilen. Sie war knapp zwei Monate lang, vom 6.5. bis 30.6.2015, online geschaltet. In diesem Zeitraum sind 1.964 auswertbare Online-Fragebögen ausgefüllt und über eine Umfrageplattform ${ }^{1}$ aufgezeichnet worden, wofür wir an dieser Stelle den zahlreichen Teilnehmer/-innen ganz herzlich danken möchten. Die Umfrage enthielt einen Gesamtbestand von 207 Fragen zu verschiedenen, lexembezogenen lautlichen Variationsphänomenen, wobei der Sitz des Wortakzents, die Quantität des betonten Vokals und Realisierungsvarianten bei Fremdwörtern die Schwerpunkte waren. Aus dem Fundus aller Fragen wurden den einzelnen Teilnehmenden jeweils 40 zufällig ausgewählte zur Beantwortung vorgelegt. Somit entfallen auf jedes abgefragte Phänomen durchschnittlich etwas weniger als $20 \%$ der gesamten Antworten, die absoluten Antwortzahlen liegen damit in der Regel zwischen $350-400$ pro Frage.

Aus Platzgründen konnte in die Druckausgabe des Duden-Aussprachewörterbuchs letztlich nur weniger als die Hälfte der Umfrageergebnisse aufgenommen werden: 91 der insgesamt 207 Fragen wurden in Form von Kastenartikeln publiziert, in der die ursprünglich in der Umfrage benutzte fünfstufige Likert-Skala (starke Zustimmung, schwache Zustimmung, neutrale Einstellung, schwache Ablehnung, starke Ablehnung) auf drei Kategorien (Zustimmung, neutral, Ablehnung) reduziert war. Da der finale Termin für die Manuskriptabgabe Anfang August 2015 nur drei Monate nach Umfragebeginn lag, musste außerdem auf einen Zwischenstand der Umfrage mit nur rund 1.000 Teilnehmenden zurückgegriffen werden. Und um „die Angemessenheitsurteile sprachräumlich auszubalancieren" (Kleiner/Knöbl/Mangold 2015, S. 16), d.h. die Antworten aus den verschiedenen Regionen Deutschlands sowie aus Österreich und der Schweiz gleichmäßig zu repräsentieren, musste daraus nochmals eine Stichprobe von nur mehr 573 Personen gebildet werden. Ausschließlich auf diesem ausgewählten guten Viertel der gesamten Teilnehmerzahl beruhen letztendlich die in der Neuauflage des Duden-Aussprachewörterbuchs publizierten Umfrageergebnisse.

Um die Umfrage sowohl hinsichtlich der Teilnehmerzahl als auch der abgefragten lautlichen Variationsphänomene in ihrem ganzen Umfang zu dokumentieren, werden im hier vorliegenden Beitrag deren Ergebnisse erstmals vollständig veröffentlicht. Die Daten werden dabei in keiner Weise modifiziert oder nach bestimmten Gesichtspunkten gewichtet oder reduziert, wodurch sich Konsequenzen für deren Auswertung ergeben, auf die im Folgenden eingegangen wird.

\section{Aufbau und Inhalt der Umfrage}

Der folgende Text wurde als Ein- und Anleitung für die Umfrage verwendet (Fettdruck im Original):

Ihnen werden immer zwei oder mehr Aussprachevarianten verschiedener Art eines bestimmten Wortes angeboten. Ihre Aufgabe ist es, die Angemessenheit dieser

Varianten in formellen Sprechsituationen zu bewerten. Formelle Sprechsituationen

1 Verwendet wurde die Unipark-Plattform (https://www.unipark.com, letzter Zugriff 12.9.2019). 
sind solche, die durch einen höheren Planungsgrad, weniger Spontanität, striktere Hierarchie oder Rollenverteilung der Gesprächspartner, mehr Öffentlichkeit und die Notwendigkeit der Wahrung gesellschaftlicher Normen charakterisiert sind.

Die Angemessenheit der Aussprachevarianten wird anhand einer 5-teiligen Skala bewertet. Neben den positiven Bewertungsoptionen „voll angemessen“ und „weitgehend angemessen“ und den negativen Entsprechungen „ganz unangemessen“ und „weitgehend unangemessen“ gibt es auch die Möglichkeit, die Varianten als „neutral“ zu bewerten.

Beachten Sie bitte auch: Die Bewertung des Angemessenheitsgrades soll unabhängig von Ihrem eigenen Sprachgebrauch in derartigen formellen Sprechsituationen erfolgen. Beurteilen Sie nur, für wie passend Sie die Varianten halten, ganz gleich, ob Sie diesem Anspruch selbst immer gerecht werden (können).

Ziel der Umfrage war also nicht, den eigenen Sprachgebrauch der Teilnehmenden zu erheben, sondern deren Bewertung für bestimmte Aussprachevarianten unabhängig vom jeweiligen eigenen Sprachgebrauch. Inwiefern dies letztendlich immer konsequent Beachtung fand, muss offen bleiben.

Die zu bewertenden Aussprachevarianten wurden in der Umfrage ausschließlich in schriftlicher Form präsentiert. Die Fragen wurden für die Teilnehmenden in vier inhaltlich zusammenhängenden Blöcken (1. Wortakzent, 2. Vokalquantität, 3. Gemischtes, 4. Lehnwörter) zusammengefasst, denen jeweils eine Erläuterung der zur Bewertung präsentierten Phänomentypen inklusive eines Beispiels vorangestellt war. ${ }^{2}$

Da jede Variante separat bewertet werden musste, kann es vorkommen, dass für ein Wort beide Varianten als mehrheitlich (voll) angemessen bewertet wurden. Dies ist besonders häufig bei den Fragen zur Vokalquantität geschehen.

Bei insgesamt 27 Wörtern, von denen angenommen wurde, dass sie einem größeren Teil der Teilnehmer/-innen unbekannt sein könnten, wurde zusätzlich danach gefragt, ob das jeweilige Wort bekannt ist, was eine gewisse Einschätzung der Zuverlässigkeit der betreffenden Angemessenheitsurteile erlaubt. Als Antwortmöglichkeiten waren nur ja oder nein zugelassen, d.h. keine Abstufungen wie in der eigentlichen Umfrage zur Bewertung der Aussprachevarianten. Die Ergebnisse sind in der nachfolgenden Tabelle 1 zusammengefasst. Die am wenigsten bekannten Wörter waren demnach die Bezeichnungen für die Holzart Bangkirai - die mit weitem Abstand auf dem letzten Platz liegt -, die Käsesorte Romadur, das Gewürz Galgant, die Bettdecke Plumeau, der Steckertyp Cinch, das Abkürzungswort aus dem Softwarebereich $C A D$ und der linguistische Fachterminus deiktisch.

2 Beispielhaft sei hier die Erläuterung zum ersten Abschnitt, in dem Variation beim Wortakzentsitz beurteilt werden sollte, angeführt (Fettdruck im Original): „Im ersten Teil sollen Sie beurteilen, welche Silbe im Wort betont werden soll, damit die Aussprache in formellen Sprechsituationen angemessen erscheint.

Die schriftliche Darstellung dabei ist folgende:

- Die betonte Silbe wird komplett großgeschrieben.

- Um diesbezüglich Unklarheiten zu vermeiden, wird auf die übliche, orthografisch geforderte Großschreibung bei Substantiven verzichtet.

Beim Wort „Roboter“ würden mögliche Antwortoptionen also folgendermaßen dargestellt:

1. Silbe betont: ROboter 2. Silbe betont: roBOter".

Die Erläuterungen der anderen, inhaltlich komplexeren Blöcke, fallen zum Teil deutlich umfangreicher aus. 


\begin{tabular}{|c|c|c|}
\hline Wort & bekannt & unbekannt \\
\hline Upgrade & 98,8 & 1,2 \\
\hline Leviten & 98,6 & 1,4 \\
\hline Fumbo & 97,4 & 2,6 \\
\hline Zip(-Datei)/gezippt & 97,1 & 2,9 \\
\hline Merlin & 96,0 & 4,0 \\
\hline Mokassin & 94,7 & 5,3 \\
\hline Sample & 93,3 & 6,7 \\
\hline Chutney & 93,6 & 6,4 \\
\hline Kardamom & 91,5 & 8,5 \\
\hline Wrigley & 90,8 & 9,2 \\
\hline Relaunch & 89,2 & 10,8 \\
\hline Mogul & 89,2 & 10,8 \\
\hline Kalmar & 85,9 & 14,1 \\
\hline Salmiak & 80,9 & 19,1 \\
\hline Obmann/frau & 79,6 & 20,4 \\
\hline Leviathan & 72,6 & 27,4 \\
\hline Milan & 71,5 & 28,5 \\
\hline Matrize & 70,3 & 29,7 \\
\hline Labskaus & 66,6 & 33,4 \\
\hline Hotel Garni & 66,2 & 33,8 \\
\hline Plumeau & 55,7 & 44,3 \\
\hline Cinch(-Stecker) & 53,1 & 46,9 \\
\hline deiktisch & 52,4 & 47,6 \\
\hline$C A D$ & 52,4 & 47,6 \\
\hline Romadur & 45,5 & 54,5 \\
\hline Galgant & 43,9 & 56,1 \\
\hline Bangkirai & 22,6 & 77,4 \\
\hline
\end{tabular}

Tabelle 1: $\quad$ (Un-)Bekannheit von 27 abgefragten Wörtern (in \%) 


\section{Zum Problem der regionalen Herkunft der Umfrage- teilnehmer/-innen}

Verschiedene der in der Umfrage zu bewertenden Aussprachevarianten zeigen im Deutschen ausgeprägte regionale Vorkommens- und Häufigkeitsunterschiede, z.B. die Aussprache von <Ch-> in China (vgl. http://prowiki.ids-mannheim.de/bin/view/AADG/Che mieCh) oder von <i> in Kredit. Es ist davon auszugehen, dass die Gebräuchlichkeit bestimmter Varianten in einzelnen Regionen einen - wohl in der Regel für deren Bewertung förderlichen - Einfluss auf die in der Umfrage erhobenen Angemessenheitsurteile für Aussprachevarianten in formellen Sprechsituationen bei solchen Teilnehmer/-innen hat, die aus den betreffenden Regionen kommen. Sollten also die Umfrageteilnehmer/-innen regionale Herkunftsschwerpunkte aufweisen, die deutlich von der allgemeinen Bevölkerungsverteilung im deutschen Sprachraum abweichen, ist entsprechend damit zu rechnen, dass davon auch die Umfrageergebnisse beeinflusst werden, dergestalt dass Varianten, die in den Herkunftsschwerpunkten üblich sind, häufiger positiv bewertet worden sein dürften.

Vorauszuschicken ist, dass wir die regionale Herkunft von 698 der insgesamt 1.964 Umfrageteilnehmer/-innen nicht kennen, weil sie darauf verzichtet haben, die entsprechenden Fragen hierzu zu beantworten. ${ }^{3}$ Die übrigen 1.266 Teilnehmer/-innen verteilen sich wie folgt auf die unterschiedlichen Regionen des deutschen Sprachraums:

\begin{tabular}{lcccc} 
Region & $\begin{array}{l}\text { Zahl der } \\
\text { Teilneh- } \\
\text { menden }\end{array}$ & $\begin{array}{l}\text { Prozent- } \\
\text { anteil } \\
\text { Teilneh- } \\
\text { mende }\end{array}$ & $\begin{array}{l}\text { Bevölke- } \\
\text { rungszahl } \\
\text { (in Mio.) }\end{array}$ & $\begin{array}{l}\text { Prozent- } \\
\text { anteil } \\
\text { Bevölke- } \\
\text { rung }\end{array}$ \\
\hline Österreich & 163 & $12,9 \%$ & 8,7 & $8,9 \%$ \\
Berlin & 33 & $2,6 \%$ & 3,6 & $3,7 \%$ \\
Baden-Württemberg & 186 & $14,7 \%$ & 11,1 & $11,3 \%$ \\
Bayern & 208 & $16,4 \%$ & 13,1 & $13,4 \%$ \\
Deutschschweiz & 83 & $6,6 \%$ & 5,3 & $5,4 \%$ \\
Brandenburg & 10 & $0,8 \%$ & 2,5 & $2,6 \%$ \\
Mecklenburg-Vorpommern & 44 & $3,5 \%$ & 1,6 & $1,6 \%$ \\
Nordrhein-Westfalen & 204 & $16,1 \%$ & 17,9 & $18,3 \%$ \\
Hessen & 34 & $2,7 \%$ & 6,3 & $6,4 \%$
\end{tabular}

3 Dass diese Zahl mehr als ein Drittel aller Teilnehmenden umfasst, ist sicher auch dem Aufbau der Umfrage zuzuschreiben: Die Daten zum Geburtsort und zur sprachlichen Selbsteinschätzung wurden nicht zu Beginn, sondern am Ende der Umfrage erhoben und es ist letztlich keine Überraschung, dass nach einer Bearbeitungszeit von durchschnittlich einer halben Stunde für die 40 sprachlichen Fragen viele Teilnehmer/-innen nicht mehr dazu motiviert werden konnten, zum Abschluss nochmals weitere Textfelder auszufüllen. 


\begin{tabular}{lcccc} 
Region & $\begin{array}{c}\text { Zahl der } \\
\text { Teilneh- } \\
\text { menden }\end{array}$ & $\begin{array}{l}\text { Prozent- } \\
\text { anteil } \\
\text { Teilneh- } \\
\text { mende }\end{array}$ & $\begin{array}{l}\text { Bevölke- } \\
\text { rungszahl } \\
\text { (in Mio.) }\end{array}$ & $\begin{array}{l}\text { Prozent- } \\
\text { anteil } \\
\text { Bevölke- } \\
\text { rung }\end{array}$ \\
\hline $\begin{array}{l}\text { Niedersachsen, Bremen, } \\
\text { Hamburg }\end{array}$ & 83 & $6,6 \%$ & 10,5 & $10,7 \%$ \\
$\begin{array}{l}\text { Rheinland-Pfalz } \\
\text { Saarland }\end{array}$ & 57 & $4,5 \%$ & 4,1 & $4,2 \%$ \\
$\begin{array}{l}\text { Schleswig-Holstein } \\
\text { Sachsen, Thüringen, }\end{array}$ & 43 & $2,2 \%$ & 1 & $1,0 \%$ \\
Sachsen-Anhalt & 73 & $5,4 \%$ & 2,9 & $3,0 \%$ \\
Luxemburg/Ostbelgien & 4 & $0,3 \%$ & 8,4 & $8,6 \%$ \\
Südtirol & 13 & $1,0 \%$ & 0,3 & $0,7 \%$ \\
Gesamt & 1.266 & $100 \%$ & 98 & $100 \%$
\end{tabular}

Tabelle 2: $\quad$ Großregionale Herkunft der Umfrageteilnehmer/-innen im Vergleich zum Bevölkerungsanteil derselben Gebiete

Man kann aufgrund der großen Anzahl von 698 Personen, die keine Angaben zum Geburtsort gemacht haben, und den damit verbundenen statistischen Wahrscheinlichkeiten davon ausgehen, dass sich diese hinsichtlich ihrer regionalen Herkunft nicht wesentlich von der in Tabelle 2 in der zweiten Spalte abgebildeten Verteilung unterscheiden, d.h. die vorhandenen Daten hierzu spiegeln die tatsächliche Verteilung der Herkunftsdaten im Gesamtkorpus ausreichend genau wider. Man wird darüber hinaus auch annehmen dürfen, dass es keine signifikanten regionalen Unterschiede hinsichtlich des Durchhaltevermögens beim Ausfüllen der Fragebögen gegeben hat. Der Vergleich der regionalen Herkunft der Umfrageteilnehmer/-innen (Spalten 1 und 2) mit den Bevölkerungsanteilen der jeweiligen Regionen (Spalten 3 und 4) zeigt, dass der Süden des deutschen Sprachraums in unserer Umfrage insgesamt um über elf Prozentpunkte stärker repräsentiert ist, als es der Bevölkerungszahl in diesen Regionen entspricht (Bayern $+3,0 \%$, Baden-Württemberg $+3,4 \%$, Österreich $+4 \%$, Deutschschweiz $+1,2 \%$ ). Umgekehrt liegen die Teilnehmerzahlen im Osten Deutschlands (ohne Mecklenburg-Vorpommern) um fünf, in der westlichen Mitte (Nordrhein-Westfalen, Hessen) um sechs und im Nordwesten um vier Prozentpunkte unter dem dortigen Bevölkerungsanteil. Allerdings gibt es auch im Norden und in der Mitte Deutschlands Regionen, die im Vergleich zu ihren Bevölkerungsanteilen adäquat (Schleswig-Holstein, Rheinland-Pfalz) oder sogar überrepräsentiert (Mecklenburg-Vorpommern, Saarland) sind.

Um einen Eindruck davon zu vermitteln, in welche Richtung die Umfrageergebnisse durch den überproportionalen Anteil von Teilnehmenden aus dem südlichen deutschen Sprachraum beeinflusst werden und damit Hinweise zur Interpretation der Rohdaten zu geben, werden in der folgenden Tabelle 3 exemplarisch sieben Beispiele aus dem für die DudenNeuauflage (Kleiner/Knöbl/Mangold 2015) regional austarierten Teildatensatz herausgegriffen und mit den ungewichteten Ergebnissen der Gesamtdaten, wie sie hier in diesem Beitrag publiziert sind, verglichen. Differenzen von $4 \%$ und höher sind durch Fettdruck hervorgehoben. 


\begin{tabular}{|c|c|c|c|}
\hline & Zustimmung & Neutral & Ablehnung \\
\hline ethnisch [e:] & $+86 \% /+84 \%$ & $6 \% / 8 \%$ & $-7 \% /-8 \%$ \\
\hline ethnisch $[\varepsilon]$ & $+42 \% /+38 \%$ & $12 \% / 14 \%$ & $-46 \% /-48 \%$ \\
\hline China $[\mathrm{c}]$ & $+82 \% /+80 \%$ & $11 \% / 10 \%$ & $-7 \% /-10 \%$ \\
\hline China $[\mathrm{k}]$ & $+38 \% /+43 \%$ & $11 \% / 11 \%$ & $-51 \% /-46 \%$ \\
\hline China $\left[\int\right]$ & $+22 \% /+22 \%$ & $18 \% / 14 \%$ & $-60 \% /-63 \%$ \\
\hline Chicorée [S] & $+75 \% /+75 \%$ & $7 \% / 8 \%$ & $-18 \% /-17 \%$ \\
\hline Chicorée $\left[\mathrm{t} \int\right]$ & $+46 \% / 44 \%$ & $16 \% / 12 \%$ & $-38 \% /-44 \%$ \\
\hline Jury [`--] & $+87 \% /+86 \%$ & $7 \% / 9 \%$ & $-6 \% /-5 \%$ \\
\hline Fury [-'- $]$ & $+39 \% /+38 \%$ & $14 \% / 15 \%$ & $-47 \% /-47 \%$ \\
\hline Kredit $[\mathrm{i}:]$ & $+71 \% /+67 \%$ & $14 \% / 11 \%$ & $-15 \% /-22 \%$ \\
\hline Kredit $[\mathrm{I}]$ & $+50 \% /+55 \%$ & $17 \% / 16 \%$ & $-33 \% /-29 \%$ \\
\hline Millimeter ['----] & $+77 \% / 78 \%$ & $11 \% / 11 \%$ & $-12 \% / 10 \%$ \\
\hline Millimeter [--'--] & $+59 \% / 58 \%$ & $25 \% / 26 \%$ & $-16 \% / 16 \%$ \\
\hline Notiz [i: $]$ & $+82 \% /+76 \%$ & $6 \% / 9 \%$ & $-12 \% /-16 \%$ \\
\hline Notiz [I] & $+41 \% /+49 \%$ & $12 \% / 16 \%$ & $-46 \% /-35 \%$ \\
\hline
\end{tabular}

Tabelle 3: Vergleich der Umfrageergebnisse; linke Prozentwerte: regional gewichteter und reduzierter Datensatz (573), rechte Prozentwerte: Gesamtdatenbestand (1.964)

Praktisch identisch fallen die Antworten der beiden verglichenen Samples bei der Bewertung der Aussprache des <e > in ethnisch als [e:], des [ç] und [J] in China, des [J] in Chicorée sowie bei der Beurteilung der Wortakzentvarianten von fury und Millimeter aus. Relevante Unterschiede gibt es hingegen für $[\varepsilon]$ in ethnisch, [k] in China sowie bei der Bewertung der Aussprache von $<\mathrm{i}>$ in Kredit und Notiz. Während für ethnisch keine Erkenntnisse zu etwaiger regionaler Variation in der Aussprache vorliegen, sind $[\mathrm{k}]$ in China ebenso wie kurzes [I] in Kredit und Notiz eindeutig Varianten, die typisch sind für den Süden des deutschen Sprachraums (zu China vgl. http://prowiki.ids-mannheim.de/ bin/view/AADG/ChemieCh). Dementsprechend lässt sich der jeweilige Anstieg der Zustimmung zu bzw. die Abnahme der Ablehnung von diesen Varianten im Gesamtdatenbestand um durchschnittlich knapp 6\% mit großer Wahrscheinlichkeit auf den erhöhten Anteil von Teilnehmer/-innen aus dem Süden des deutschen Sprachraums zurückführen. Allerdings ist die Spannbreite von Fall zu Fall erheblich und der Effekt kann sogar - wie bei Millimeter, wo der Akzenttyp ['----] ebenfalls eher im Süden gebräuchlich ist - auch ganz ausbleiben. 


\section{Die Falktoren Geschlecht, Alter, Schulbildung und Beruf}

Hinsichtlich des Geschlechts sind die Umfrageteilnehmer/-innen weit von einer repräsentativen, d.h. in diesem Fall ungefähr gleichmäßigen Verteilung auf beide Geschlechter, entfernt: 904 Frauen, aber nur 393 Männer haben teilgenommen. Inwiefern aus diesem Missverhältnis der Geschlechter ebenfalls Einflüsse auf die Bewertungen der Aussprachevarianten entstanden sein könnten, muss offen bleiben; ein gegebenenfalls vorhandener Einfluss dürfte jedoch sicher weit geringer ausfallen als derjenige der regionalen Herkunft.

Das Alter der Teilnehmer/-innen wurde ebenfalls erhoben, eine Verteilung auf die unterschiedlichen Altersgruppen ist in Abbildung 1 zusammengefasst.

$49 \%$ von ihnen waren zum Zeitpunkt der Erhebung jünger als 31 Jahre, $20 \%$ jünger als 41 , $15 \%$ jünger als $51,11 \%$ jünger als $61,4 \%$ jünger als 71 und nur knapp $2 \%$ von ihnen mehr als 71 Jahre alt. Die Altersstruktur der Umfrageteilnehmer/-innen ist mit ihrem Schwerpunkt auf der jüngeren Generation einerseits typisch für eine online durchgeführte Umfrage, andererseits spiegelt sich in ihr wohl auch eine wesentliche Rekrutierungsstrategie, die vor allem die sozialen Netzwerke der Projektmitarbeiter/-innen (fünf davon im Alter von 22-35 Jahren) als Multiplikatoren nutzte.

Auch der Bildungsgrad wurde mit den üblichen Fragen nach dem höchsten Schulabschluss erhoben, 94\% haben Hochschulreife, 53\% einen Hochschulabschluss. Außerdem wurde nach dem derzeit oder früher ausgeübten Beruf bzw. der laufenden Ausbildung gefragt, die in einem freien Textfeld angegeben werden konnten. Es wurde hier keine exakte Auswertung vorgenommen, aber - entsprechend den bereits genannten Zahlen zur Schulbildung - ca. 90\% der genannten Berufe bzw. der laufenden Ausbildungen sind akademischer Natur, und von diesen stammt wiederum die Mehrheit aus dem sprachlich-philologischpädagogischen Bereich.

Alter der Umfrageteilnehmer/-innen

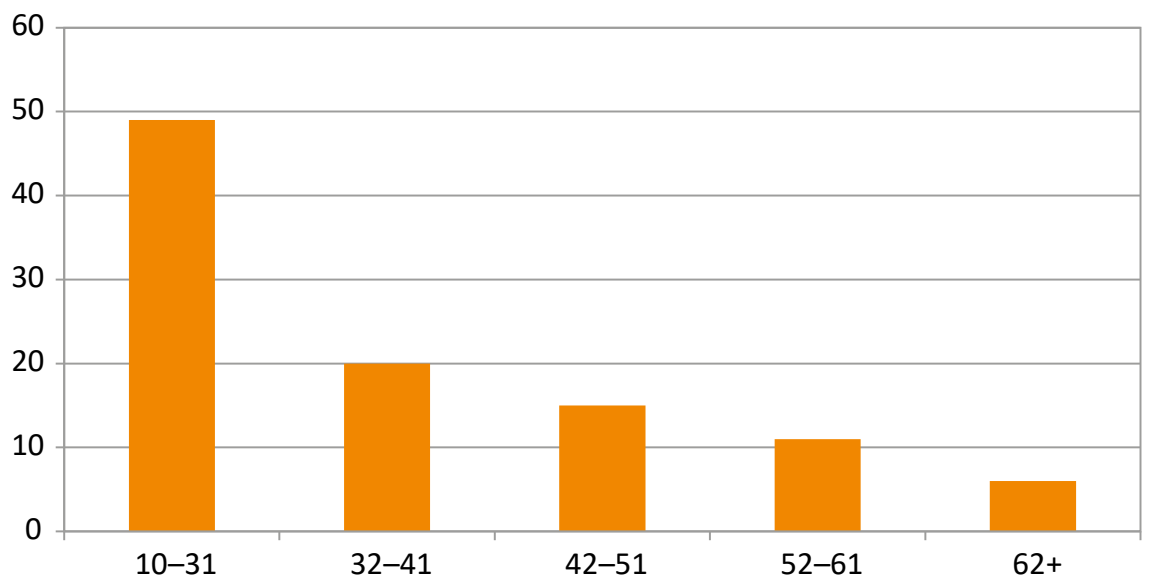

Abbildung 1: Altersstruktur der Umfrageteilnehmer/-innen 


\section{Die Umfrageergebnisse}

\subsection{Teil 1: Betonte Silbe}

Im ersten Teil der Umfrage, der 51 Fragen umfasst, sollte die Angemessenheit von Wortakzentvarianten beurteilt werden. Die Varianten werden wie in der Umfrage dargestellt: Die jeweils betonte Silbe ist großgeschrieben, die nicht betonten klein. Alle Zahlenangaben in den folgenden Tabellen sind Prozentwerte.

\begin{tabular}{l|l|l} 
& ANIS & aNIS \\
\cline { 2 - 3 } & Anis & 48,25 \\
\hline voll angemessen & 42,11 & 12,87 \\
\hline weitgehend angemessen & 11,40 & 8,77 \\
\hline neutral & 10,23 & 12,57 \\
\hline weitgehend unangemessen & 14,62 & 17,54
\end{tabular}

\begin{tabular}{l|l|l|l} 
& \multicolumn{2}{l}{ BANGKIRAI } \\
\cline { 2 - 4 } & BANGkirai & bangKIrai & bangkiRAI \\
\hline voll angemessen & 35,29 & 8,24 & 18,82 \\
\hline weitgehend angemessen & 27,06 & 14,12 & 31,76 \\
\hline neutral & 18,82 & 14,12 & 17,65 \\
\hline weitgehend unangemessen & 12,94 & 30,59 & 21,18 \\
\hline ganz unangemessen & 5,88 & 32,94 & 10,59
\end{tabular}

\begin{tabular}{l|l|l} 
& \multicolumn{2}{|l}{ BEATRIX (Name) } \\
\cline { 2 - 3 } & BEatrix & beAtrix \\
\hline voll angemessen & 70,98 & 14,87 \\
\hline weitgehend angemessen & 15,11 & 10,31 \\
\hline neutral & 6,95 & 13,19 \\
\hline weitgehend unangemessen & 4,56 & 27,10 \\
\hline ganz unangemessen & 2,40 & 34,53
\end{tabular}




\begin{tabular}{l|l|l} 
& \multicolumn{2}{|l}{ BONIFAZ (Name) } \\
\cline { 2 - 3 } & BOnifaz & boniFAZ \\
\hline voll angemessen & 39,32 & 31,51 \\
\hline weitgehend angemessen & 20,83 & 16,15 \\
\hline neutral & 16,41 & 16,67 \\
\hline weitgehend unangemessen & 14,32 & 22,40 \\
\hline ganz unangemessen & 9,11 & 13,28
\end{tabular}

\begin{tabular}{|c|c|c|}
\hline & \multicolumn{2}{|c|}{ CABRIOLET } \\
\hline & CAbriolet & cabrioLET \\
\hline voll angemessen & 52,20 & 29,12 \\
\hline weitgehend angemessen & 20,60 & 17,86 \\
\hline neutral & 12,91 & 19,51 \\
\hline weitgehend unangemessen & 10,16 & 18,68 \\
\hline ganz unangemessen & 4,12 & 14,84 \\
\hline
\end{tabular}

\begin{tabular}{l|l|l} 
& CARAVAN & caraVAN \\
\cline { 2 - 3 } & CAravan & 8,97 \\
\hline voll angemessen & 68,60 & 11,35 \\
\hline weitgehend angemessen & 17,68 & 22,96 \\
\hline neutral & 7,39 & 32,72 \\
\hline weitgehend unangemessen & 4,22 & 24,01
\end{tabular}

\begin{tabular}{|c|c|c|}
\hline & \multicolumn{2}{|c|}{ DIAKON } \\
\hline & DIAkon & diaKON \\
\hline voll angemessen & 37,97 & 46,40 \\
\hline weitgehend angemessen & 14,14 & 15,14 \\
\hline neutral & 15,14 & 10,17 \\
\hline weitgehend unangemessen & 17,37 & 17,37 \\
\hline ganz unangemessen & 15,38 & 10,92 \\
\hline
\end{tabular}




\begin{tabular}{l|l|l} 
& GALGANT & galGANT \\
\cline { 2 - 3 } & GALgant & 49,70 \\
\hline voll angemessen & 25,44 & 17,75 \\
\hline weitgehend angemessen & 13,02 & 17,75 \\
\hline neutral & 18,93 & 10,65 \\
\hline weitgehend unangemessen & 23,08 & 4,14
\end{tabular}

\begin{tabular}{l|l|l} 
& GIGABYTE & gigaBYTE \\
\cline { 2 - 3 } & GIgabyte & 6,23 \\
\hline voll angemessen & 78,50 & 7,01 \\
\hline weitgehend angemessen & 11,66 & 15,32 \\
\hline neutral & 6,48 & 32,99 \\
\hline weitgehend unangemessen & 2,07 & 38,44
\end{tabular}

\begin{tabular}{l|l|l} 
& \multicolumn{2}{|l}{ HOTEL GARNI } \\
\cline { 2 - 3 } & (Hotel) GARni & (Hotel) garNI \\
\hline voll angemessen & 53,21 & 26,52 \\
\hline weitgehend angemessen & 13,21 & 9,85 \\
\hline neutral & 9,43 & 12,50 \\
\hline weitgehend unangemessen & 9,43 & 19,32 \\
\hline ganz unangemessen & 14,72 & 31,82
\end{tabular}

\begin{tabular}{|c|c|c|}
\hline & \multicolumn{2}{|c|}{ IMPOTENT } \\
\hline & IMpotent & impoTENT \\
\hline voll angemessen & 84,22 & 5,09 \\
\hline weitgehend angemessen & 10,43 & 3,56 \\
\hline neutral & 1,78 & 9,92 \\
\hline weitgehend unangemessen & 2,04 & 30,79 \\
\hline ganz unangemessen & 1,53 & 50,64 \\
\hline
\end{tabular}




\begin{tabular}{|c|c|c|}
\hline & \multicolumn{2}{|c|}{ INAKZEPTABEL } \\
\hline & INakzeptabel & inakzepTAbel \\
\hline voll angemessen & 85,64 & 7,18 \\
\hline weitgehend angemessen & 9,90 & 13,37 \\
\hline neutral & 2,23 & 15,59 \\
\hline weitgehend unangemessen & 1,49 & 34,65 \\
\hline \multirow[t]{3}{*}{ ganz unangemessen } & 0,74 & 29,21 \\
\hline & \multicolumn{2}{|l|}{ KAKADU } \\
\hline & KAkadu & kakaDU \\
\hline voll angemessen & 70,55 & 11,95 \\
\hline weitgehend angemessen & 14,29 & 10,5 \\
\hline neutral & 4,66 & 15,16 \\
\hline weitgehend unangemessen & 5,54 & 29,74 \\
\hline ganz unangemessen & 4,96 & 32,65 \\
\hline
\end{tabular}

\begin{tabular}{|c|c|c|}
\hline & \multicolumn{2}{|c|}{ KALMAR } \\
\hline & KALmar & kalMAR \\
\hline voll angemessen & 9,66 & 65,34 \\
\hline weitgehend angemessen & 11,08 & 16,48 \\
\hline neutral & 16,48 & 8,52 \\
\hline weitgehend unangemessen & 33,81 & 6,53 \\
\hline ganz unangemessen & 28,98 & 3,12 \\
\hline
\end{tabular}

\begin{tabular}{l|l|l} 
& \multicolumn{2}{|l}{ KARDAMOM } \\
\cline { 2 - 3 } & KARdamom & kardaMOM \\
\hline voll angemessen & 79,51 & 3,81 \\
\hline weitgehend angemessen & 13,11 & 7,36 \\
\hline neutral & 4,10 & 13,08 \\
\hline weitgehend unangemessen & 1,91 & 35,69 \\
\hline ganz unangemessen & 1,37 & 40,05
\end{tabular}




\begin{tabular}{|c|c|c|}
\hline & \multicolumn{2}{|c|}{ KARDANWELLE } \\
\hline & KARdan(welle) & karDAN(welle) \\
\hline voll angemessen & 45,67 & 9,19 \\
\hline weitgehend angemessen & 18,37 & 12,34 \\
\hline neutral & 26,51 & 35,17 \\
\hline weitgehend unangemessen & 6,04 & 20,73 \\
\hline \multirow[t]{3}{*}{ ganz unangemessen } & 3,41 & 22,57 \\
\hline & \multicolumn{2}{|l|}{ KILOMETER } \\
\hline & KIlometer & kiloMEter \\
\hline voll angemessen & 37,05 & 53,27 \\
\hline weitgehend angemessen & 22,28 & 19,61 \\
\hline neutral & 16,71 & 10,90 \\
\hline weitgehend unangemessen & 15,01 & 9,93 \\
\hline ganz unangemessen & 8,96 & 6,30 \\
\hline
\end{tabular}

\begin{tabular}{l|l|l|l} 
& \multicolumn{2}{l}{ KIMONO } & kiMOno \\
\cline { 2 - 4 } & KImono & 7,63 & 4,33 \\
\hline voll angemessen & 73,54 & 9,67 & 6,11 \\
\hline weitgehend angemessen & 12,72 & 16,28 & 15,27 \\
\hline neutral & 5,60 & 31,81 & 29,26 \\
\hline weitgehend unangemessen & 5,34 & 34,61 & 45,04
\end{tabular}

\begin{tabular}{|c|c|c|}
\hline & \multicolumn{2}{|c|}{ KNOW-HOW } \\
\hline & KNOW-how & know-HOW \\
\hline voll angemessen & 23,29 & 61,01 \\
\hline weitgehend angemessen & 12,91 & 16,46 \\
\hline neutral & 18,23 & 11,14 \\
\hline weitgehend unangemessen & 25,57 & 5,82 \\
\hline ganz unangemessen & 20,00 & 5,57 \\
\hline
\end{tabular}




\begin{tabular}{|c|c|c|}
\hline & \multicolumn{2}{|l|}{ KONVOI } \\
\hline & KONvoi & konVOI \\
\hline voll angemessen & 35,56 & 47,65 \\
\hline weitgehend angemessen & 22,47 & 22,72 \\
\hline neutral & 18,77 & 14,07 \\
\hline weitgehend unangemessen & 16,54 & 10,86 \\
\hline \multirow[t]{3}{*}{ ganz unangemessen } & 6,67 & 4,69 \\
\hline & \multicolumn{2}{|c|}{ LEUKOPLAST } \\
\hline & LEUkoplast & leukoPLAST \\
\hline voll angemessen & 24,44 & 53,87 \\
\hline weitgehend angemessen & 18,45 & 18,20 \\
\hline neutral & 22,69 & 12,97 \\
\hline weitgehend unangemessen & 21,45 & 10,97 \\
\hline ganz unangemessen & 12,97 & 3,99 \\
\hline
\end{tabular}

\begin{tabular}{l|l|l|l} 
& \multicolumn{2}{l}{ LEVIATHAN } \\
\cline { 2 - 4 } & LEviathan & leviaTHAN & leviAthan \\
\hline voll angemessen & 16,34 & 36,63 & 13,12 \\
\hline weitgehend angemessen & 19,06 & 16,34 & 14,85 \\
\hline neutral & 22,77 & 21,53 & 18,07 \\
\hline weitgehend unangemessen & 22,77 & 14,85 & 23,02 \\
\hline ganz unangemessen & 19,06 & 10,64 & 30,94
\end{tabular}

\begin{tabular}{|c|c|c|}
\hline & \multicolumn{2}{|c|}{ LIEBKOSEN } \\
\hline & LIEBkosen & liebKOsen \\
\hline voll angemessen & 11,17 & 72,99 \\
\hline weitgehend angemessen & 10,91 & 14,29 \\
\hline neutral & 15,58 & 4,94 \\
\hline weitgehend unangemessen & 37,14 & 5,19 \\
\hline ganz unangemessen & 25,19 & 2,60 \\
\hline
\end{tabular}




\begin{tabular}{l|l|l} 
& \multicolumn{2}{|l}{ LIESELOTTE (Name) } \\
\cline { 2 - 3 } & LIEselotte & lieseLOTte \\
\hline voll angemessen & 32,80 & 48,15 \\
\hline weitgehend angemessen & 16,67 & 19,58 \\
\hline neutral & 21,69 & 11,90 \\
\hline weitgehend unangemessen & 20,37 & 13,23 \\
\hline ganz unangemessen & 8,47 & 7,14
\end{tabular}

\begin{tabular}{l|l|l} 
& \multicolumn{2}{l}{ LUTHERISCH } \\
\cline { 2 - 3 } & LUtherisch & luTHErisch \\
\hline voll angemessen & 57,80 & 18,41 \\
\hline weitgehend angemessen & 17,39 & 16,62 \\
\hline neutral & 9,72 & 13,55 \\
\hline weitgehend unangemessen & 8,70 & 20,97 \\
\hline ganz unangemessen & 6,39 & 30,43
\end{tabular}

\begin{tabular}{l|l|l} 
& MAJORAN & majoRAN \\
\cline { 2 - 3 } & MAjoran & 5,00 \\
\hline voll angemessen & 82,04 & 6,50 \\
\hline weitgehend angemessen & 11,72 & 10,25 \\
\hline neutral & 4,24 & 37,75 \\
\hline weitgehend unangemessen & 2,00 & 40,50
\end{tabular}

\begin{tabular}{|c|c|c|}
\hline & \multicolumn{2}{|l|}{ MAKE-UP } \\
\hline & MAKE-up & make-UP \\
\hline voll angemessen & 62,86 & 26,75 \\
\hline weitgehend angemessen & 21,82 & 23,12 \\
\hline neutral & 7,79 & 17,14 \\
\hline weitgehend unangemessen & 4,94 & 20,00 \\
\hline ganz unangemessen & 2,60 & 12,99 \\
\hline
\end{tabular}




\begin{tabular}{l|l|l} 
& MASSAKER & masSAker \\
\cline { 2 - 3 } & MASsaker & 40,25 \\
\hline voll angemessen & 45,82 & 24,30 \\
\hline weitgehend angemessen & 20,51 & 15,70 \\
\hline neutral & 13,42 & 13,42 \\
\hline weitgehend unangemessen & 10,89 & 6,33
\end{tabular}

\begin{tabular}{|c|c|c|}
\hline & \multicolumn{2}{|c|}{ MATHEMATIK } \\
\hline & matheMAtik & mathemaTIK \\
\hline voll angemessen & 14,68 & 70,38 \\
\hline weitgehend angemessen & 15,44 & 15,95 \\
\hline neutral & 11,39 & 5,32 \\
\hline weitgehend unangemessen & 29,11 & 3,29 \\
\hline ganz unangemessen & 29,37 & 5,06 \\
\hline
\end{tabular}

\begin{tabular}{l|l|l} 
& \multicolumn{2}{|l}{ MEGAWATT } \\
\cline { 2 - 3 } & MEgawatt & megaWATT \\
\hline voll angemessen & 77,08 & 7,81 \\
\hline weitgehend angemessen & 11,72 & 8,85 \\
\hline neutral & 4,95 & 15,36 \\
\hline weitgehend unangemessen & 2,86 & 35,16 \\
\hline ganz unangemessen & 3,39 & 32,81
\end{tabular}

\begin{tabular}{l|l|l|l} 
& \multicolumn{2}{|l}{ MELANIE (Name) } \\
\cline { 2 - 4 } & MElanie & melaNIE & meLANie \\
\hline voll angemessen & 74,52 & 10,80 & 2,77 \\
\hline weitgehend angemessen & 10,53 & 13,57 & 3,88 \\
\hline neutral & 5,54 & 16,34 & 8,59 \\
\hline weitgehend unangemessen & 5,82 & 27,70 & 12,47 \\
\hline ganz unangemessen & 3,60 & 31,58 & 72,30
\end{tabular}




\begin{tabular}{|c|c|c|}
\hline & \multicolumn{2}{|c|}{ MENOPAUSE } \\
\hline & MEnopause & menoPAUse \\
\hline voll angemessen & 77,18 & 7,95 \\
\hline weitgehend angemessen & 13,33 & 3,85 \\
\hline neutral & 5,64 & 15,64 \\
\hline weitgehend unangemessen & 2,31 & 34,10 \\
\hline ganz unangemessen & 1,54 & 38,46 \\
\hline
\end{tabular}

\begin{tabular}{l|l|l} 
& \multicolumn{2}{|l}{ MERKUR } \\
\cline { 2 - 3 } & MERkur & merKUR \\
\hline voll angemessen & 59,54 & 24,17 \\
\hline weitgehend angemessen & 20,10 & 18,32 \\
\hline neutral & 9,92 & 22,39 \\
\hline weitgehend unangemessen & 8,40 & 19,34 \\
\hline ganz unangemessen & 2,04 & 15,78
\end{tabular}

\begin{tabular}{|c|c|c|}
\hline & \multicolumn{2}{|c|}{ MERLIN } \\
\hline & MERlin & merLIN \\
\hline voll angemessen & 85,92 & 4,79 \\
\hline weitgehend angemessen & 7,04 & 5,92 \\
\hline neutral & 4,51 & 11,27 \\
\hline weitgehend unangemessen & 1,41 & 27,32 \\
\hline ganz unangemessen & 1,13 & 50,70 \\
\hline
\end{tabular}

\begin{tabular}{l|l|l} 
& \multicolumn{2}{|l}{ MIKROGRAMM } \\
\cline { 2 - 3 } & MIkrogramm & mikroGRAMM \\
\hline voll angemessen & 73,10 & 6,85 \\
\hline weitgehend angemessen & 15,99 & 9,64 \\
\hline neutral & 5,58 & 18,53 \\
\hline weitgehend unangemessen & 3,05 & 31,22 \\
\hline ganz unangemessen & 2,28 & 33,76
\end{tabular}




\begin{tabular}{l|l|l} 
& MILAN & miLAN \\
\cline { 2 - 3 } & MIlan & 44,57 \\
\hline voll angemessen & 35,87 & 14,13 \\
\hline weitgehend angemessen & 13,77 & 13,41 \\
\hline neutral & 18,48 & 15,94 \\
\hline weitgehend unangemessen & 19,20 & 11,96
\end{tabular}

\begin{tabular}{l|l|l} 
& \multicolumn{2}{|l}{ MILLIMETER } \\
\cline { 2 - 3 } & MILlimeter & milliMEter \\
\hline voll angemessen & 58,49 & 36,66 \\
\hline weitgehend angemessen & 17,79 & 21,56 \\
\hline neutral & 11,32 & 15,90 \\
\hline weitgehend unangemessen & 8,36 & 15,90 \\
\hline ganz unangemessen & 4,04 & 9,97
\end{tabular}

\begin{tabular}{l|l|l} 
& \multicolumn{2}{|l}{ MISSACHTUNG } \\
\cline { 2 - 3 } & MISSachtung & missACHtung \\
\hline voll angemessen & 36,82 & 53,23 \\
\hline weitgehend angemessen & 21,64 & 20,90 \\
\hline neutral & 14,18 & 7,96 \\
\hline weitgehend unangemessen & 15,42 & 10,45 \\
\hline ganz unangemessen & 11,94 & 7,46
\end{tabular}

\begin{tabular}{|c|c|c|}
\hline & \multicolumn{2}{|c|}{ MOGUL } \\
\hline & MOgul & moGUL \\
\hline voll angemessen & 10,85 & 76,98 \\
\hline weitgehend angemessen & 8,99 & 11,90 \\
\hline neutral & 9,52 & 4,23 \\
\hline weitgehend unangemessen & 30,95 & 4,76 \\
\hline ganz unangemessen & 39,68 & 2,12 \\
\hline
\end{tabular}




\begin{tabular}{l|l|l} 
& MOKASSIN & mokasSIN \\
\cline { 2 - 3 } voll angemessen & MOkassin & 13,37 \\
\hline weitgehend angemessen & 65,04 & 11,31 \\
\hline neutral & 13,88 & 13,88 \\
\hline weitgehend unangemessen & 5,91 & 32,39 \\
\hline ganz unangemessen & 9,00 & 29,05 \\
\hline \multirow{2}{*}{ voll angemessen } & 6,17 & \\
\hline weitgehend angemessen & NEPTUN & nepTUN \\
\hline neutral & NEPtun & 9,18 \\
\hline weitgehend unangemessen & 80,60 & 6,95 \\
\hline ganz unangemessen & 1,24 & 19,11 \\
\hline & 1,00 & 27,30 \\
\hline
\end{tabular}

\begin{tabular}{l|l|l} 
& \multicolumn{2}{|l}{ PANKRAZ (Name) } \\
\cline { 2 - 3 } & PANkraz & panKRAZ \\
\hline voll angemessen & 54,32 & 8,40 \\
\hline weitgehend angemessen & 21,48 & 7,90 \\
\hline neutral & 18,02 & 28,89 \\
\hline weitgehend unangemessen & 4,69 & 31,60 \\
\hline ganz unangemessen & 1,48 & 23,21
\end{tabular}

\begin{tabular}{|c|c|c|}
\hline & \multicolumn{2}{|c|}{ PLUMEAU } \\
\hline & PLUmeau & pluMEAU \\
\hline voll angemessen & 21,33 & 64,44 \\
\hline weitgehend angemessen & 12,00 & 10,67 \\
\hline neutral & 12,00 & 4,89 \\
\hline weitgehend unangemessen & 25,33 & 12,00 \\
\hline ganz unangemessen & 29,33 & 8,00 \\
\hline
\end{tabular}




\begin{tabular}{l|l|l} 
& \multicolumn{2}{l}{ REBOOT } \\
\cline { 2 - 3 } & REboot & reBOOT \\
\hline voll angemessen & 41,26 & 28,88 \\
\hline weitgehend angemessen & 17,96 & 16,99 \\
\hline neutral & 21,12 & 24,51 \\
\hline weitgehend unangemessen & 11,41 & 17,96 \\
\hline ganz unangemessen & 8,25 & 11,65
\end{tabular}

\begin{tabular}{l|l|l} 
& RESET & reSET \\
\cline { 2 - 3 } & REset & 53,38 \\
\hline voll angemessen & 33,10 & 18,41 \\
\hline weitgehend angemessen & 20,98 & 13,29 \\
\hline neutral & 14,92 & 10,02 \\
\hline weitgehend unangemessen & 16,08 & 4,90
\end{tabular}

\begin{tabular}{|c|c|c|}
\hline & \multicolumn{2}{|c|}{ ROMADUR } \\
\hline & ROmadur & romaDUR \\
\hline voll angemessen & 52,54 & 29,94 \\
\hline weitgehend angemessen & 15,25 & 15,82 \\
\hline neutral & 15,25 & 10,17 \\
\hline weitgehend unangemessen & 12,43 & 20,90 \\
\hline ganz unangemessen & 4,52 & 23,16 \\
\hline
\end{tabular}

\begin{tabular}{|c|c|c|}
\hline & \multicolumn{2}{|c|}{ SALMIAK } \\
\hline & SALmiak & salMIAK \\
\hline voll angemessen & 69,67 & 15,32 \\
\hline weitgehend angemessen & 13,51 & 9,31 \\
\hline neutral & 4,80 & 10,51 \\
\hline weitgehend unangemessen & 8,11 & 30,93 \\
\hline ganz unangemessen & 3,90 & 33,93 \\
\hline
\end{tabular}




\begin{tabular}{l|l|l} 
& \multicolumn{2}{l}{ TELENOVELA } \\
\cline { 2 - 3 } & TElenovela & telenoVEla \\
\hline voll angemessen & 22,81 & 53,70 \\
\hline weitgehend angemessen & 17,51 & 20,90 \\
\hline neutral & 22,81 & 8,73 \\
\hline weitgehend unangemessen & 19,89 & 9,26 \\
\hline ganz unangemessen & 16,98 & 7,41
\end{tabular}

\begin{tabular}{|c|c|c|}
\hline & \multicolumn{2}{|c|}{ WYOMING } \\
\hline & WYoming & wyOming \\
\hline voll angemessen & 16,63 & 60,39 \\
\hline weitgehend angemessen & 14,43 & 17,60 \\
\hline neutral & 23,23 & 10,02 \\
\hline weitgehend unangemessen & 22,74 & 7,58 \\
\hline ganz unangemessen & 22,98 & 4,40 \\
\hline
\end{tabular}

\begin{tabular}{l|l|l} 
& YAMAHA & yaMAha \\
\cline { 2 - 3 } & YAmaha & 61,77 \\
\hline voll angemessen & 15,44 & 21,52 \\
\hline weitgehend angemessen & 11,14 & 9,62 \\
\hline neutral & 22,53 & 4,81 \\
\hline weitgehend unangemessen & 26,33 & 2,28
\end{tabular}

\begin{tabular}{l|l|l} 
& ZITRONAT & zitroNAT \\
\cline { 2 - 3 } & ZItronat & 57,54 \\
\hline voll angemessen & 25,06 & 14,32 \\
\hline weitgehend angemessen & 13,04 & 10,49 \\
\hline neutral & 19,44 & 8,44 \\
\hline weitgehend unangemessen & 21,74 & 9,21
\end{tabular}




\subsection{Teil 2: Vokalquantitåt}

Die Varianten der 49 Wörter aus dem zweiten Teil der Umfrage unterscheiden sich immer in Bezug auf die Quantität des Vokals der betonten Silbe, der „lang“ oder „kurz“ ausgesprochen werden kann, wodurch sich bei der Mehrheit der deutschen Muttersprachler/-innen (Ausnahmen v.a. in Österreich, Schweiz und Südwestdeutschland) in der Regel auch die Vokalqualität verändert: Die kurzen Varianten werden, außer bei <a>, auch offener und mit zentralisierterer Zungenposition ausgesprochen. In der linken Spalte sind jeweils die kurzvokalischen Varianten aufgeführt, in der rechten die langvokalischen, bei denen die Vokallänge zusätzlich mit einem nachgestellten Doppelpunkt markiert wird. Dieselbe diakritische Markierung wurde auch in der Umfrage verwendet, zur Verdeutlichung wurde in dieser auch der Kurzvokal mit einem Bogen unter dem Vokal eigens markiert (z. B. „Arzt“). Außerdem wurden in der Umfrage die thematisierten Vokale in den Varianten ergänzend rot eingefärbt und dadurch zusätzlich hervorgehoben.

\begin{tabular}{l|l|l} 
& AFTER & A:fter \\
\cline { 2 - 3 } & After & 10,67 \\
\hline voll angemessen & 29,60 & 10,42 \\
\hline weitgehend angemessen & 17,91 & 9,18 \\
\hline neutral & 18,91 & 17,37 \\
\hline weitgehend unangemessen & 19,90 & 52,36
\end{tabular}

\begin{tabular}{l|l|l} 
& ARTIKEL & Arti:kel \\
\cline { 2 - 3 } & Artikel & 28,93 \\
\hline voll angemessen & 61,19 & 17,71 \\
\hline weitgehend angemessen & 15,92 & 13,97 \\
\hline neutral & 9,95 & 17,96 \\
\hline weitgehend unangemessen & 8,71 & 21,45
\end{tabular}

\begin{tabular}{l|l|l} 
& ARZT & A:rzt \\
\cline { 2 - 3 } & Arzt & 57,60 \\
\hline voll angemessen & 29,41 & 14,46 \\
\hline weitgehend angemessen & 11,76 & 13,48 \\
\hline neutral & 20,59 & 9,31 \\
\hline weitgehend unangemessen & 18,87 & 5,15
\end{tabular}




\begin{tabular}{l|l|l} 
& BARSCH (Fisch) \\
\cline { 2 - 3 } & Barsch & Ba:rsch \\
\hline voll angemessen & 42,12 & 36,21 \\
\hline weitgehend angemessen & 15,76 & 19,21 \\
\hline neutral & 18,23 & 14,53 \\
\hline weitgehend unangemessen & 14,04 & 12,56 \\
\hline ganz unangemessen & 9,85 & 17,49
\end{tabular}

\begin{tabular}{l|l|l} 
& \multicolumn{2}{|l}{ BARSCH (unfreundlich) } \\
\cline { 2 - 3 } & barsch & ba:rsch \\
\hline voll angemessen & 57,46 & 25,18 \\
\hline weitgehend angemessen & 15,40 & 11,74 \\
\hline neutral & 10,02 & 17,36 \\
\hline weitgehend unangemessen & 10,76 & 20,78 \\
\hline ganz unangemessen & 6,36 & 24,94
\end{tabular}

\begin{tabular}{l|l|l} 
& \multicolumn{2}{|l}{ BETABLOCKER } \\
\cline { 2 - 3 } & Be्tablocker & Be:tablocker \\
\hline voll angemessen & 41,73 & 42,34 \\
\hline weitgehend angemessen & 19,90 & 21,77 \\
\hline neutral & 11,51 & 11,96 \\
\hline weitgehend unangemessen & 16,31 & 12,20 \\
\hline ganz unangemessen & 10,55 & 11,72
\end{tabular}

\begin{tabular}{|c|c|c|}
\hline & \multicolumn{2}{|c|}{ DAMWILD } \\
\hline & Damwild & Da:mwild \\
\hline voll angemessen & 64,95 & 12,66 \\
\hline weitgehend angemessen & 12,11 & 7,49 \\
\hline neutral & 12,63 & 15,25 \\
\hline weitgehend unangemessen & 6,44 & 28,42 \\
\hline ganz unangemessen & 3,87 & 36,18 \\
\hline
\end{tabular}




\begin{tabular}{|c|c|c|}
\hline & \multicolumn{2}{|c|}{ DESASTER } \\
\hline & Desåster & Desa:ster \\
\hline voll angemessen & 10,14 & 78,39 \\
\hline weitgehend angemessen & 8,99 & 12,18 \\
\hline neutral & 15,21 & 3,45 \\
\hline weitgehend unangemessen & 34,10 & 3,68 \\
\hline \multirow[t]{3}{*}{ ganz unangemessen } & 31,57 & 2,30 \\
\hline & \multicolumn{2}{|l|}{ EDITOR } \\
\hline & Editor & E:ditor \\
\hline voll angemessen & 56,79 & 22,85 \\
\hline weitgehend angemessen & 18,55 & 18,55 \\
\hline neutral & 12,67 & 19,00 \\
\hline weitgehend unangemessen & 6,79 & 20,81 \\
\hline ganz unangemessen & 5,20 & 18,78 \\
\hline
\end{tabular}

\begin{tabular}{l|l|l} 
& ERZ & E:rz \\
\cline { 2 - 3 } & Erz & 29,51 \\
\hline voll angemessen & 53,76 & 13,35 \\
\hline weitgehend angemessen & 13,62 & 16,16 \\
\hline neutral & 12,21 & 22,72 \\
\hline weitgehend unangemessen & 13,15 & 18,27
\end{tabular}

\begin{tabular}{l|l|l} 
& \multicolumn{2}{l}{ ERZBISCHOF } \\
\cline { 2 - 3 } & Erzbischof & E:rzbischof \\
\hline voll angemessen & 58,92 & 26,76 \\
\hline weitgehend angemessen & 16,43 & 14,79 \\
\hline neutral & 11,03 & 19,01 \\
\hline weitgehend unangemessen & 8,45 & 21,36 \\
\hline ganz unangemessen & 5,16 & 18,08
\end{tabular}




\begin{tabular}{l|l|l} 
& ESTRICH & E:strich \\
\cline { 2 - 3 } & Estrich & 14,14 \\
\hline voll angemessen & 74,94 & 8,93 \\
\hline weitgehend angemessen & 12,41 & 15,38 \\
\hline neutral & 5,96 & 29,28 \\
\hline weitgehend unangemessen & 2,98 & 32,26
\end{tabular}

\begin{tabular}{|c|c|c|}
\hline & \multicolumn{2}{|c|}{ EST(H)ER } \\
\hline & Est(h)er & E:st(h)er \\
\hline voll angemessen & 45,72 & 50,61 \\
\hline weitgehend angemessen & 19,80 & 13,94 \\
\hline neutral & 18,09 & 13,69 \\
\hline weitgehend unangemessen & 11,74 & 11,49 \\
\hline ganz unangemessen & 4,65 & 10,27 \\
\hline
\end{tabular}

\begin{tabular}{|c|c|c|}
\hline & \multicolumn{2}{|c|}{ ETHNISCH } \\
\hline & enthnisch & e:thnisch \\
\hline voll angemessen & 20,67 & 66,07 \\
\hline weitgehend angemessen & 17,08 & 18,20 \\
\hline neutral & 14,38 & 7,64 \\
\hline weitgehend unangemessen & 27,87 & 5,62 \\
\hline ganz unangemessen & 20,00 & 2,47 \\
\hline
\end{tabular}

\begin{tabular}{l|l|l} 
& FABRIK & Fabri:k \\
\cline { 2 - 3 } & Fabrik & 37,53 \\
\hline voll angemessen & 53,01 & 18,16 \\
\hline weitgehend angemessen & 19,76 & 12,11 \\
\hline neutral & 9,88 & 16,22 \\
\hline weitgehend unangemessen & 10,12 & 15,98
\end{tabular}




\begin{tabular}{l|l|l} 
& FLORIDA & Flo:rida \\
\cline { 2 - 3 } & Florida & 9,29 \\
\hline voll angemessen & 75,59 & 11,67 \\
\hline weitgehend angemessen & 16,11 & 13,57 \\
\hline neutral & 4,03 & 35,48 \\
\hline weitgehend unangemessen & 1,90 & 30,00
\end{tabular}

\begin{tabular}{|c|c|c|}
\hline & \multicolumn{2}{|c|}{ GESTE } \\
\hline & Genste & Ge:ste \\
\hline voll angemessen & 7,53 & 82,82 \\
\hline weitgehend angemessen & 4,94 & 10,59 \\
\hline neutral & 6,35 & 3,29 \\
\hline weitgehend unangemessen & 28,24 & 1,18 \\
\hline ganz unangemessen & 52,94 & 2,12 \\
\hline
\end{tabular}

\begin{tabular}{|c|c|c|}
\hline & \multicolumn{2}{|c|}{ GESTIK } \\
\hline & Gestik & Ge:stik \\
\hline voll angemessen & 11,29 & 76,24 \\
\hline weitgehend angemessen & 7,06 & 12,47 \\
\hline neutral & 14,35 & 4,47 \\
\hline weitgehend unangemessen & 29,88 & 3,06 \\
\hline ganz unangemessen & 37,41 & 3,76 \\
\hline
\end{tabular}

\begin{tabular}{|c|c|c|}
\hline & \multicolumn{2}{|l|}{ GIBT } \\
\hline & gibt & gi:bt \\
\hline voll angemessen & 48,42 & 45,26 \\
\hline weitgehend angemessen & 19,22 & 18,73 \\
\hline neutral & 15,57 & 13,14 \\
\hline weitgehend unangemessen & 11,44 & 12,65 \\
\hline ganz unangemessen & 5,35 & 10,22 \\
\hline
\end{tabular}




\begin{tabular}{l|l|l} 
& GING & gi:ng \\
\cline { 2 - 3 } & ging & 23,75 \\
\hline voll angemessen & 72,04 & 7,84 \\
\hline weitgehend angemessen & 8,77 & 5,46 \\
\hline neutral & 5,69 & 22,09 \\
\hline weitgehend unangemessen & 8,53 & 40,86
\end{tabular}

\begin{tabular}{l|l|l} 
& GRANIT & Grani:t \\
\cline { 2 - 3 } & Granit & 46,15 \\
\hline voll angemessen & 41,16 & 15,85 \\
\hline weitgehend angemessen & 17,21 & 11,89 \\
\hline neutral & 13,72 & 13,29 \\
\hline weitgehend unangemessen & 18,37 & 12,82
\end{tabular}

\begin{tabular}{l|l|l} 
& HINAUF & hi:nauf \\
\cline { 2 - 3 } & hinauf & 26,75 \\
\hline voll angemessen & 61,20 & 10,12 \\
\hline weitgehend angemessen & 13,49 & 10,60 \\
\hline neutral & 10,36 & 23,61 \\
\hline weitgehend unangemessen & 8,92 & 28,92
\end{tabular}

\begin{tabular}{l|l|l} 
& ISRAEL & I:srael \\
\cline { 2 - 3 } & Israel & 69,31 \\
\hline voll angemessen & 17,82 & 17,82 \\
\hline weitgehend angemessen & 10,64 & 3,47 \\
\hline neutral & 13,37 & 4,95 \\
\hline weitgehend unangemessen & 33,42 & 4,46
\end{tabular}




\begin{tabular}{|c|c|c|}
\hline & \multicolumn{2}{|l|}{ JAKOB } \\
\hline & Jakob & Ja:kob \\
\hline voll angemessen & 25,25 & 64,36 \\
\hline weitgehend angemessen & 16,09 & 16,09 \\
\hline neutral & 17,57 & 9,16 \\
\hline weitgehend unangemessen & 23,02 & 6,19 \\
\hline \multirow[t]{3}{*}{ ganz unangemessen } & 18,07 & 4,21 \\
\hline & \multicolumn{2}{|c|}{ JAN (Name) } \\
\hline & Jan & Ja:n \\
\hline voll angemessen & 65,71 & 33,81 \\
\hline weitgehend angemessen & 10,55 & 14,87 \\
\hline neutral & 13,91 & 17,27 \\
\hline weitgehend unangemessen & 6,24 & 19,66 \\
\hline \multirow[t]{3}{*}{ ganz unangemessen } & 3,60 & 14,39 \\
\hline & \multicolumn{2}{|c|}{ JOSEPHA } \\
\hline & Josepha & Jose:pha \\
\hline voll angemessen & 62,53 & 15,75 \\
\hline weitgehend angemessen & 15,15 & 10,77 \\
\hline neutral & 9,37 & 17,40 \\
\hline weitgehend unangemessen & 8,54 & 26,24 \\
\hline ganz unangemessen & 4,41 & 29,83 \\
\hline
\end{tabular}

\begin{tabular}{l|l|l} 
& JUPITER & Ju:piter \\
\cline { 2 - 3 } & Jụpiter & 47,66 \\
\hline voll angemessen & 39,81 & 19,86 \\
\hline weitgehend angemessen & 22,72 & 14,95 \\
\hline neutral & 13,82 & 10,98 \\
\hline weitgehend unangemessen & 14,52 & 6,54
\end{tabular}




\begin{tabular}{l|l|l} 
& KAPUZE & Kapu:ze \\
\cline { 2 - 3 } & Kapuze & 58,85 \\
\hline voll angemessen & 27,51 & 20,33 \\
\hline weitgehend angemessen & 14,59 & 6,46 \\
\hline neutral & 16,03 & 8,37 \\
\hline weitgehend unangemessen & 22,01 & 5,98
\end{tabular}

\begin{tabular}{l|l|l} 
& KONSUL & Konsu:l \\
\cline { 2 - 3 } & Konsul & 45,89 \\
\hline voll angemessen & 35,75 & 19,32 \\
\hline weitgehend angemessen & 16,18 & 11,35 \\
\hline neutral & 12,56 & 14,01 \\
\hline weitgehend unangemessen & 17,39 & 9,42
\end{tabular}

\begin{tabular}{l|l|l} 
& KREDIT & Kredi:t \\
\cline { 2 - 3 } & Kredit & 52,31 \\
\hline voll angemessen & 35,22 & 14,87 \\
\hline weitgehend angemessen & 20,05 & 10,51 \\
\hline neutral & 15,68 & 11,03 \\
\hline weitgehend unangemessen & 17,48 & 11,28
\end{tabular}

\begin{tabular}{|c|c|c|}
\hline & \multicolumn{2}{|c|}{ LABSKAUS } \\
\hline & Labskaus & La:bskaus \\
\hline voll angemessen & 71,27 & 15,52 \\
\hline weitgehend angemessen & 9,45 & 6,14 \\
\hline neutral & 8,36 & 13,36 \\
\hline weitgehend unangemessen & 6,55 & 22,38 \\
\hline ganz unangemessen & 4,36 & 42,60 \\
\hline
\end{tabular}




\begin{tabular}{l|l|l} 
& \multicolumn{2}{|l}{ LATSCHE (Schuh) } \\
\cline { 2 - 3 } & Latsche & La:tsche \\
\hline voll angemessen & 3,33 & 82,23 \\
\hline weitgehend angemessen & 2,85 & 11,14 \\
\hline neutral & 7,84 & 2,13 \\
\hline weitgehend unangemessen & 26,37 & 1,90 \\
\hline ganz unangemessen & 59,62 & 2,61
\end{tabular}

\begin{tabular}{l|l|l} 
& \multicolumn{2}{|l}{ LATSCHE (Kiefernart) } \\
\cline { 2 - 3 } & Latsche & La:tsche \\
\hline voll angemessen & 7,86 & 53,33 \\
\hline weitgehend angemessen & 9,29 & 13,57 \\
\hline neutral & 28,33 & 23,81 \\
\hline weitgehend unangemessen & 15,00 & 6,19 \\
\hline ganz unangemessen & 39,52 & 3,10
\end{tabular}

\begin{tabular}{|c|c|c|}
\hline & \multicolumn{2}{|c|}{ LEVITEN } \\
\hline & Leviten & Levi:ten \\
\hline voll angemessen & 20,67 & 65,87 \\
\hline weitgehend angemessen & 12,74 & 14,42 \\
\hline neutral & 10,82 & 6,49 \\
\hline weitgehend unangemessen & 27,88 & 6,97 \\
\hline ganz unangemessen & 27,88 & 6,25 \\
\hline
\end{tabular}

\begin{tabular}{l|l|l} 
& LITAUEN & Li:tauen \\
\cline { 2 - 3 } & Litauen & 4,56 \\
\hline voll angemessen & 86,11 & 5,57 \\
\hline weitgehend angemessen & 9,60 & 10,63 \\
\hline neutral & 2,53 & 32,66 \\
\hline weitgehend unangemessen & 0,76 & 46,58
\end{tabular}




\begin{tabular}{l|l|l} 
& \multicolumn{2}{|l}{ MAKROKOSMOS } \\
\cline { 2 - 3 } & Mąrokosmos & Ma:krokosmos \\
\hline voll angemessen & 48,93 & 32,86 \\
\hline weitgehend angemessen & 17,81 & 14,05 \\
\hline neutral & 11,40 & 12,38 \\
\hline weitgehend unangemessen & 14,73 & 23,33 \\
\hline ganz unangemessen & 7,13 & 17,38
\end{tabular}

\begin{tabular}{l|l|l} 
& MATRIZE & Matri:ze \\
\cline { 2 - 3 } & Matrize & 8,11 \\
\hline voll angemessen & 76,43 & 3,04 \\
\hline weitgehend angemessen & 13,80 & 8,78 \\
\hline neutral & 7,41 & 32,09 \\
\hline weitgehend unangemessen & 1,68 & 47,97
\end{tabular}

\begin{tabular}{l|l|l} 
& NISCHE & Ni:sche \\
\cline { 2 - 3 } & Nische & 84,50 \\
\hline voll angemessen & 7,50 & 9,00 \\
\hline weitgehend angemessen & 3,25 & 1,50 \\
\hline neutral & 2,50 & 2,00 \\
\hline weitgehend unangemessen & 22,25 & 3,00
\end{tabular}

\begin{tabular}{l|l|l} 
& NOTIZ & Noti:z \\
\cline { 2 - 3 } & Notiz & 58,63 \\
\hline voll angemessen & 32,62 & 17,02 \\
\hline weitgehend angemessen & 16,55 & 8,51 \\
\hline neutral & 15,84 & 9,46 \\
\hline weitgehend unangemessen & 18,86 & 6,38
\end{tabular}




\begin{tabular}{l|l|l} 
& \multicolumn{2}{l}{ OBMANN/-FRAU } \\
\cline { 2 - 3 } & Obmann/-frau & O:bmann/-frau \\
\hline voll angemessen & 71,34 & 16,77 \\
\hline weitgehend angemessen & 12,50 & 7,32 \\
\hline neutral & 5,49 & 11,89 \\
\hline weitgehend unangemessen & 6,71 & 25,30 \\
\hline ganz unangemessen & 3,96 & 38,72
\end{tabular}

\begin{tabular}{|c|c|c|}
\hline & \multicolumn{2}{|c|}{ OMEGA } \\
\hline & Omega & O:mega \\
\hline voll angemessen & 80,65 & 12,90 \\
\hline weitgehend angemessen & 11,17 & 11,91 \\
\hline neutral & 3,97 & 16,87 \\
\hline weitgehend unangemessen & 2,48 & 29,78 \\
\hline ganz unangemessen & 1,74 & 28,54 \\
\hline
\end{tabular}

\begin{tabular}{l|l|l} 
& PARTIKEL & Parti:kel \\
\cline { 2 - 3 } & Partikel & 24,11 \\
\hline voll angemessen & 63,59 & 15,60 \\
\hline weitgehend angemessen & 19,86 & 14,42 \\
\hline neutral & 6,62 & 21,28 \\
\hline weitgehend unangemessen & 6,15 & 24,59
\end{tabular}

\begin{tabular}{l|l|l} 
& RELATIV & re:lativ \\
\cline { 2 - 3 } & ręlativ & 20,87 \\
\hline voll angemessen & 69,25 & 23,06 \\
\hline weitgehend angemessen & 17,92 & 16,99 \\
\hline neutral & 9,20 & 24,03 \\
\hline weitgehend unangemessen & 2,66 & 15,05
\end{tabular}




\begin{tabular}{|c|c|c|}
\hline & \multicolumn{2}{|c|}{ REPUBLIK } \\
\hline & Republik & Republi:k \\
\hline voll angemessen & 50,13 & 44,05 \\
\hline weitgehend angemessen & 18,33 & 16,76 \\
\hline neutral & 14,02 & 11,08 \\
\hline weitgehend unangemessen & 11,05 & 13,78 \\
\hline \multirow[t]{3}{*}{ ganz unangemessen } & 6,47 & 14,32 \\
\hline & \multicolumn{2}{|c|}{ SATELLIT } \\
\hline & Satellit & Satelli:t \\
\hline voll angemessen & 33,49 & 54,50 \\
\hline weitgehend angemessen & 18,53 & 17,77 \\
\hline neutral & 15,44 & 5,69 \\
\hline weitgehend unangemessen & 21,62 & 11,37 \\
\hline ganz unangemessen & 10,93 & 10,66 \\
\hline
\end{tabular}

\begin{tabular}{|c|c|c|}
\hline & \multicolumn{2}{|c|}{ SPIRITUS } \\
\hline & Spiritus & Spirritus \\
\hline voll angemessen & 54,33 & 33,13 \\
\hline weitgehend angemessen & 16,72 & 16,42 \\
\hline neutral & 11,04 & 11,34 \\
\hline weitgehend unangemessen & 11,94 & 25,07 \\
\hline ganz unangemessen & 5,97 & 14,03 \\
\hline
\end{tabular}

\begin{tabular}{|c|c|c|}
\hline & \multicolumn{2}{|c|}{ UNERFAHREN } \\
\hline & unnerfahren & u:nerfahren \\
\hline voll angemessen & 75,54 & 13,61 \\
\hline weitgehend angemessen & 13,20 & 6,70 \\
\hline neutral & 3,90 & 11,66 \\
\hline weitgehend unangemessen & 4,98 & 27,00 \\
\hline ganz unangemessen & 2,38 & 41,04 \\
\hline
\end{tabular}




\begin{tabular}{|c|c|c|}
\hline & \multicolumn{2}{|c|}{ WALNUSS } \\
\hline & Wạlnuss & Wa:lnuss \\
\hline voll angemessen & 40,86 & 47,85 \\
\hline weitgehend angemessen & 14,47 & 12,66 \\
\hline neutral & 5,84 & 8,35 \\
\hline weitgehend unangemessen & 17,26 & 11,90 \\
\hline ganz unangemessen & 21,57 & 19,24 \\
\hline
\end{tabular}

\begin{tabular}{l|l|l} 
& WALROSS & Wa:lross \\
\cline { 2 - 3 } & Wạlross & 84,20 \\
\hline voll angemessen & 7,33 & 9,67 \\
\hline weitgehend angemessen & 4,73 & 3,30 \\
\hline neutral & 8,04 & 1,89 \\
\hline weitgehend unangemessen & 27,42 & 0,94
\end{tabular}

\subsection{Teil 3: Gemischte Phänomene}

In diesem Teil der Umfrage wurden Aussprachevarianten unterschiedlicher Art abgefragt. Sie wurden im Folgenden zu thematisch zusammengehörigen Gruppen zusammengefasst. In der Darstellung wird Lautschrift (geschrieben in eckigen Klammern) verwendet, deren Bedeutung in den voranstehenden kurzen Einleitungstexten an Beispielwörtern erläutert wird (Vokalqualitäten sind die der überregionalen bundesdeutschen Standardaussprache).

Die folgenden zwölf Wörter beginnen alle mit $<$ St-/st-> oder $<$ Sp-/sp- $>$. Die zur Wahl gestellten Aussprachevarianten waren $s c h t-/ s c h p$ - oder $s t-/ s p$-. In phonetischer Umschrift wird im Folgenden sch als [J] und $s$ als [s] wiedergegeben. Die [J]-Variante befindet sich jeweils in der linken Spalte.

\begin{tabular}{|c|c|c|}
\hline & \multicolumn{2}{|c|}{ SPIRITUS } \\
\hline & [S]piritus & [s]piritus \\
\hline voll angemessen & 75,83 & 18,84 \\
\hline weitgehend angemessen & 13,29 & 13,07 \\
\hline neutral & 6,04 & 17,33 \\
\hline weitgehend unangemessen & 3,02 & 23,71 \\
\hline ganz unangemessen & 1,81 & 27,05 \\
\hline
\end{tabular}




\begin{tabular}{l|l|l} 
& \multicolumn{2}{l}{ SPOILER (Fahrzeugteil) } \\
\cline { 2 - 3 } & [S]poiler & [s]poiler \\
\hline voll angemessen & 41,18 & 41,30 \\
\hline weitgehend angemessen & 15,59 & 12,98 \\
\hline neutral & 11,76 & 15,63 \\
\hline weitgehend unangemessen & 15,00 & 15,63 \\
\hline ganz unangemessen & 16,47 & 14,45
\end{tabular}

\begin{tabular}{l|l|l} 
& \multicolumn{2}{|l}{ SPOILER (Handlungspreisgabe) } \\
\cline { 2 - 3 } & [S]poiler & {$[$ s]poiler } \\
\hline voll angemessen & 5,01 & 76,70 \\
\hline weitgehend angemessen & 6,78 & 8,26 \\
\hline neutral & 14,16 & 10,32 \\
\hline weitgehend unangemessen & 22,12 & 2,06 \\
\hline ganz unangemessen & 51,92 & 2,65
\end{tabular}

\begin{tabular}{|c|c|c|}
\hline & \multicolumn{2}{|c|}{ STABIL } \\
\hline & {$\left[\int\right]$ tabil } & [s]tabil \\
\hline voll angemessen & 86,50 & 9,97 \\
\hline weitgehend angemessen & 9,00 & 10,29 \\
\hline neutral & 2,57 & 18,97 \\
\hline weitgehend unangemessen & 0,64 & 31,19 \\
\hline ganz unangemessen & 1,29 & 29,58 \\
\hline
\end{tabular}

\begin{tabular}{|c|c|c|}
\hline & \multicolumn{2}{|c|}{ INSTABIL } \\
\hline & in $\left[\int\right]$ tabil & in $[\mathrm{s}]$ tabil \\
\hline voll angemessen & 72,35 & 20,90 \\
\hline weitgehend angemessen & 14,15 & 17,68 \\
\hline neutral & 6,11 & 18,97 \\
\hline weitgehend unangemessen & 5,14 & 24,44 \\
\hline ganz unangemessen & 2,25 & 18,01 \\
\hline
\end{tabular}




\begin{tabular}{|c|c|c|}
\hline & \multicolumn{2}{|c|}{ STATISTIK } \\
\hline & [J]tatistik & [s]tatistik \\
\hline voll angemessen & 78,55 & 20,63 \\
\hline weitgehend angemessen & 12,93 & 10,48 \\
\hline neutral & 2,84 & 22,22 \\
\hline weitgehend unangemessen & 2,52 & 27,94 \\
\hline \multirow[t]{3}{*}{ ganz unangemessen } & 3,15 & 18,73 \\
\hline & \multicolumn{2}{|l|}{ STEAK } \\
\hline & [S]teak & {$[\mathrm{s}] \mathrm{teak}$} \\
\hline voll angemessen & 10,65 & 87,32 \\
\hline weitgehend angemessen & 11,83 & 6,49 \\
\hline neutral & 7,10 & 2,95 \\
\hline weitgehend unangemessen & 27,51 & 1,47 \\
\hline ganz unangemessen & 42,90 & 1,77 \\
\hline
\end{tabular}

\begin{tabular}{|c|c|c|}
\hline & \multicolumn{2}{|l|}{ STEREO } \\
\hline & {$\left[\int\right]$ tereo } & {$[\mathrm{s}]$ tereo } \\
\hline voll angemessen & 34,19 & 55,48 \\
\hline weitgehend angemessen & 17,74 & 17,42 \\
\hline neutral & 11,94 & 12,90 \\
\hline weitgehend unangemessen & 18,71 & 9,35 \\
\hline ganz unangemessen & 17,42 & 4,84 \\
\hline
\end{tabular}

\begin{tabular}{|c|c|c|}
\hline & \multicolumn{2}{|l|}{ STIL } \\
\hline & {$\left[\int\right]$ til } & {$[\mathrm{s}] \mathrm{til}$} \\
\hline voll angemessen & 22,55 & 78,80 \\
\hline weitgehend angemessen & 10,87 & 8,97 \\
\hline neutral & 10,05 & 5,71 \\
\hline weitgehend unangemessen & 25,82 & 4,89 \\
\hline ganz unangemessen & 30,71 & 1,63 \\
\hline
\end{tabular}




\begin{tabular}{|c|c|c|}
\hline & \multicolumn{2}{|c|}{ STILISTISCH } \\
\hline & {$\left[\int\right]$ tilistisch } & [s]tilistisch \\
\hline voll angemessen & 18,75 & 78,80 \\
\hline weitgehend angemessen & 12,77 & 10,60 \\
\hline neutral & 12,77 & 6,52 \\
\hline weitgehend unangemessen & 25,82 & 3,26 \\
\hline ganz unangemessen & 29,89 & 0,82 \\
\hline
\end{tabular}

\begin{tabular}{|c|c|c|}
\hline & \multicolumn{2}{|c|}{ STRIPTEASE } \\
\hline & {$\left[\int\right]$ triptease } & {$[\mathrm{s}]$ triptease } \\
\hline voll angemessen & 10,00 & 82,05 \\
\hline weitgehend angemessen & 6,00 & 7,98 \\
\hline neutral & 5,14 & 4,56 \\
\hline weitgehend unangemessen & 26,29 & 3,70 \\
\hline ganz unangemessen & 52,57 & 1,71 \\
\hline
\end{tabular}

\begin{tabular}{l|l|l} 
& \multicolumn{2}{l}{ STRUKTUR } \\
\cline { 2 - 3 } & []]truktur & {$[$ s]truktur } \\
\hline voll angemessen & 73,72 & 20,91 \\
\hline weitgehend angemessen & 15,71 & 17,58 \\
\hline neutral & 4,23 & 19,39 \\
\hline weitgehend unangemessen & 4,23 & 21,21 \\
\hline ganz unangemessen & 2,11 & 20,91
\end{tabular}

Die folgenden acht Wörter beginnen mit dem bzw. enthalten den Buchstaben $<\mathrm{V} / \mathrm{v}>$. Die jeweiligen Varianten sind die Aussprache wie $<\mathrm{w}>([\mathrm{v}])$ oder wie $<\mathrm{f}>([\mathrm{f}])$.

\begin{tabular}{|c|c|c|}
\hline & \multicolumn{2}{|c|}{ VENTIL } \\
\hline & {$[\mathrm{v}]$ entil } & [f]entil \\
\hline voll angemessen & 78,02 & 13,26 \\
\hline weitgehend angemessen & 10,44 & 7,18 \\
\hline neutral & 1,92 & 7,18 \\
\hline weitgehend unangemessen & 3,02 & 23,48 \\
\hline ganz unangemessen & 6,59 & 48,90 \\
\hline
\end{tabular}




\begin{tabular}{|c|c|c|}
\hline & \multicolumn{2}{|l|}{ VERS } \\
\hline & {$[v]$ ers } & [f]ers \\
\hline voll angemessen & 8,12 & 86,55 \\
\hline weitgehend angemessen & 3,75 & 5,90 \\
\hline neutral & 5,94 & 1,55 \\
\hline weitgehend unangemessen & 13,12 & 4,04 \\
\hline \multirow[t]{3}{*}{ ganz unangemessen } & 69,06 & 1,86 \\
\hline & \multicolumn{2}{|c|}{ VITUS (Name) } \\
\hline & [v]itus & [f]itus \\
\hline voll angemessen & 67,81 & 14,25 \\
\hline weitgehend angemessen & 14,25 & 7,69 \\
\hline neutral & 8,83 & 17,66 \\
\hline weitgehend unangemessen & 4,56 & 23,93 \\
\hline ganz unangemessen & 4,56 & 36,47 \\
\hline
\end{tabular}

\begin{tabular}{l|l|l} 
& \multicolumn{2}{|l}{ ALTERNATIVE } \\
\cline { 2 - 3 } & Alternati[v]e & Alternati[f]e \\
\hline voll angemessen & 61,40 & 27,13 \\
\hline weitgehend angemessen & 11,55 & 8,84 \\
\hline neutral & 6,69 & 7,01 \\
\hline weitgehend unangemessen & 7,60 & 17,68 \\
\hline ganz unangemessen & 12,77 & 39,33
\end{tabular}

\begin{tabular}{l|l|l} 
& \multicolumn{2}{|l}{ HANNOVERANER } \\
\cline { 2 - 3 } & Hanno[v]eraner & Hanno[f]eraner \\
\hline voll angemessen & 46,53 & 38,33 \\
\hline weitgehend angemessen & 21,10 & 18,16 \\
\hline neutral & 14,16 & 17,87 \\
\hline weitgehend unangemessen & 8,96 & 15,56 \\
\hline ganz unangemessen & 9,25 & 10,09
\end{tabular}




\begin{tabular}{l|l|l} 
& \multicolumn{2}{l}{ JEVER (Ortsname) } \\
\cline { 2 - 3 } & Je[v]er & Je[f]er \\
\hline voll angemessen & 68,40 & 10,77 \\
\hline weitgehend angemessen & 14,11 & 6,15 \\
\hline neutral & 11,96 & 19,38 \\
\hline weitgehend unangemessen & 3,07 & 22,77 \\
\hline ganz unangemessen & 2,45 & 40,92
\end{tabular}

\begin{tabular}{l|l|l} 
& \multicolumn{2}{l}{ XAVER (Name) } \\
\cline { 2 - 3 } & Xa[v]er & Xa[f]er \\
\hline voll angemessen & 60,44 & 23,99 \\
\hline weitgehend angemessen & 16,82 & 14,33 \\
\hline neutral & 12,15 & 15,26 \\
\hline weitgehend unangemessen & 6,23 & 20,56 \\
\hline ganz unangemessen & 4,36 & 25,86
\end{tabular}

Die folgenden vier Wörter enden auf bzw. enthalten unbetontes $<-$ ig $>$. Die jeweils abgefragten Varianten waren $-i c h[c]$, $-i k[\mathrm{k}]$ oder $-i g[\mathrm{~g}]$.

\begin{tabular}{l|l|l|l} 
& \multicolumn{2}{l}{ KÖNIG } & Köni[g] \\
\cline { 2 - 4 } & Köni[ç] & Könik] & 55,21 \\
\hline voll angemessen & 44,70 & 33,24 & 16,15 \\
\hline weitgehend angemessen & 15,47 & 17,48 & 8,85 \\
\hline neutral & 10,03 & 12,03 & 10,42 \\
\hline weitgehend unangemessen & 13,47 & 13,47 & 9,38
\end{tabular}

\begin{tabular}{l|l|l|l} 
& \multicolumn{2}{l}{ RICHTIG } & richti[g] \\
\cline { 2 - 4 } & richti[ç] & richti[k] & 50,79 \\
\hline voll angemessen & 43,40 & 35,38 & 16,23 \\
\hline weitgehend angemessen & 16,72 & 17,84 & 14,14 \\
\hline neutral & 10,56 & 14,33 & 5,24 \\
\hline weitgehend unangemessen & 12,02 & 13,74 & 13,61
\end{tabular}




\begin{tabular}{l|l|l|l} 
& \multicolumn{2}{l}{ WENIGSTENS } \\
\cline { 2 - 4 } & weni[ç]stens & weni[k]stens & weni[g]stens \\
\hline voll angemessen & 18,40 & 40,49 & 76,47 \\
\hline weitgehend angemessen & 15,95 & 18,40 & 9,63 \\
\hline neutral & 13,19 & 13,80 & 5,88 \\
\hline weitgehend unangemessen & 24,54 & 14,11 & 2,67 \\
\hline ganz unangemessen & 27,91 & 13,19 & 5,35
\end{tabular}

\begin{tabular}{l|l|l|l} 
& \multicolumn{2}{l}{ ZWANZIG } & zwanzi[g] \\
\cline { 2 - 4 } & zwanzi[ç] & zwanzi[k] & 46,24 \\
\hline voll angemessen & 43,32 & 35,01 & 20,81 \\
\hline weitgehend angemessen & 19,29 & 19,88 & 12,14 \\
\hline neutral & 11,87 & 16,62 & 9,25 \\
\hline weitgehend unangemessen & 11,87 & 13,65 & 11,56
\end{tabular}

Die folgenden drei Wörter beginnen mit der Graphie $<\mathrm{Ch}->$. Die zur Auswahl gestellten Varianten sind die Aussprache als $k$ [k], sch [J] oder $c h$ [c]].

\begin{tabular}{l|l|l|l} 
& \multicolumn{2}{l}{ CHEMIE } & [ [ ] ]emie \\
\cline { 2 - 4 } & {$[$ k]emie } & 13,11 & 67,48 \\
\hline voll angemessen & 29,88 & 15,24 & 13,68 \\
\hline weitgehend angemessen & 13,11 & 9,45 & 7,90 \\
\hline neutral & 8,23 & 20,12 & 6,69 \\
\hline weitgehend unangemessen & 18,60 & 42,07 & 4,26
\end{tabular}

\begin{tabular}{|c|c|c|c|}
\hline & \multicolumn{3}{|c|}{ CHINA } \\
\hline & {$[\mathrm{k}]$ ina } & [S]ina & [ç]ina \\
\hline voll angemessen & 31,42 & 9,64 & 64,65 \\
\hline weitgehend angemessen & 11,78 & 12,35 & 15,71 \\
\hline neutral & 11,18 & 14,46 & 9,67 \\
\hline weitgehend unangemessen & 20,24 & 25,60 & 4,23 \\
\hline ganz unangemessen & 25,38 & 37,95 & 5,74 \\
\hline
\end{tabular}




\begin{tabular}{l|l|l|l} 
& \multicolumn{2}{l}{ CHIRURG } & [ç]irurg \\
\cline { 2 - 4 } & {$[$ k]irurg } & []]irurg & 62,31 \\
\hline voll angemessen & 15,50 & 28,10 & 17,93 \\
\hline weitgehend angemessen & 14,59 & 18,13 & 8,51 \\
\hline neutral & 12,16 & 12,99 & 6,38 \\
\hline weitgehend unangemessen & 13,37 & 15,71 & 4,86
\end{tabular}

Auch bei Chicoree geht es um die Aussprache von $<$ Ch->. Die Varianten waren $s c h\left[\int\right]$ und $t s c h[\mathrm{t}]$.

\begin{tabular}{|c|c|c|}
\hline & \multicolumn{2}{|c|}{ CHICOREE } \\
\hline & [S]icoree & {$[\mathrm{t} f]$ icoree } \\
\hline voll angemessen & 54,78 & 31,95 \\
\hline weitgehend angemessen & 20,70 & 11,82 \\
\hline neutral & 7,96 & 12,14 \\
\hline weitgehend unangemessen & 10,19 & 20,45 \\
\hline ganz unangemessen & 6,37 & 23,64 \\
\hline
\end{tabular}

Bei Zucchini geht es um die Aussprache von $<\mathrm{cch}>$. Die möglichen Varianten waren $k[\mathrm{k}]$ und $c h[\mathrm{x}]$ (wie in Dach).

\begin{tabular}{|c|c|c|}
\hline & \multicolumn{2}{|c|}{ ZUCCHINI } \\
\hline & $\mathrm{Zu}[\mathrm{k}]$ ini & $\mathrm{Zu}[\mathrm{x}]$ ini \\
\hline voll angemessen & 70,83 & 29,85 \\
\hline weitgehend angemessen & 12,20 & 14,63 \\
\hline neutral & 8,04 & 10,75 \\
\hline weitgehend unangemessen & 5,06 & 16,72 \\
\hline ganz unangemessen & 3,87 & 28,06 \\
\hline
\end{tabular}


Die folgenden fünf Wörter enthalten alle den Buchstaben $<y>$, der hier entweder ungerundet als $[\mathrm{i}(\mathrm{)})]$ oder gerundet als $[\mathrm{y}(\mathrm{:})]$ realisiert werden kann.

\begin{tabular}{l|l|l} 
& ZYLINDER & Z[y]linder \\
\cline { 2 - 3 } & Z[i]linder & 28,76 \\
\hline voll angemessen & 56,72 & 19,89 \\
\hline weitgehend angemessen & 18,28 & 13,98 \\
\hline neutral & 11,29 & 20,70 \\
\hline weitgehend unangemessen & 9,41 & 16,67 \\
\hline ganz unangemessen & 4,30 & \\
\hline voll angemessen & POLYGAMIE & Pol[y]gamie \\
\hline weitgehend angemessen & Pol[i]gamie & 3,38 \\
\hline neutral & 89,85 & 3,08 \\
\hline weitgehend unangemessen & 1,23 & 8,92 \\
\hline ganz unangemessen & 0,62 & 21,85 \\
\hline
\end{tabular}

\begin{tabular}{l|l|l} 
& POLYNESIEN & Pol[y]nesien \\
\cline { 2 - 3 } & Pol[i]nesien & 3,85 \\
\hline voll angemessen & 86,69 & 6,21 \\
\hline weitgehend angemessen & 8,28 & 10,95 \\
\hline neutral & 2,96 & 26,33 \\
\hline weitgehend unangemessen & 1,18 & 52,66
\end{tabular}

\begin{tabular}{|c|c|c|}
\hline & \multicolumn{2}{|l|}{ EMBRYO } \\
\hline & Embr $[\mathrm{i}] \mathrm{o}$ & Embr[y]o \\
\hline \multicolumn{3}{|l|}{ voll angemessen } \\
\hline weitgehend angemessen & 12,09 & 6,80 \\
\hline neutral & 3,24 & 13,02 \\
\hline weitgehend unangemessen & 1,47 & 24,56 \\
\hline ganz unangemessen & 0,88 & 50,59 \\
\hline
\end{tabular}




\begin{tabular}{l|l|l} 
& FORSYTHIE & Fors[y:]thie \\
\cline { 2 - 3 } & Fors[i] thie & 27,91 \\
\hline voll angemessen & 50,29 & 13,66 \\
\hline weitgehend angemessen & 13,95 & 15,41 \\
\hline neutral & 15,70 & 18,60 \\
\hline weitgehend unangemessen & 12,21 & 24,42
\end{tabular}

Die folgenden vier Wörter enthalten den Buchstaben $<$ ä>, der hier nach traditionellen Aussprachekonventionen als offenes [ $\varepsilon$ : ] zu sprechen ist. Die möglichen Varianten waren geschlossenes langes [e:] (wie in See) und offenes langes [ع:] (wie in fett, aber lang).

\begin{tabular}{l|l|l} 
& MÄDCHEN & M[ع:]dchen \\
\cline { 2 - 3 } & M[e:]dchen & 56,09 \\
\hline voll angemessen & 41,21 & 18,59 \\
\hline weitgehend angemessen & 22,04 & 12,18 \\
\hline neutral & 15,34 & 8,01 \\
\hline weitgehend unangemessen & 13,10 & 5,13
\end{tabular}

\begin{tabular}{|c|c|c|}
\hline & \multicolumn{2}{|l|}{ ZÄHNE } \\
\hline & Z[e:]hne & $Z[\varepsilon:]$ hne \\
\hline voll angemessen & 27,22 & 66,77 \\
\hline weitgehend angemessen & 19,82 & 17,21 \\
\hline neutral & 15,38 & 8,61 \\
\hline weitgehend unangemessen & 25,15 & 5,04 \\
\hline ganz unangemessen & 12,43 & 2,37 \\
\hline
\end{tabular}

\begin{tabular}{l|l|l} 
& KÄSE & K[є:]se \\
\cline { 2 - 3 } & K[e:]se & 64,69 \\
\hline voll angemessen & 36,28 & 16,02 \\
\hline weitgehend angemessen & 18,58 & 7,42 \\
\hline neutral & 16,52 & 8,01 \\
\hline weitgehend unangemessen & 16,81 & 3,86
\end{tabular}




\begin{tabular}{l|l|l} 
& SÄGEN & s[घ:]gen \\
\cline { 2 - 3 } & s[e:]gen & 69,60 \\
\hline voll angemessen & 26,44 & 14,29 \\
\hline weitgehend angemessen & 15,81 & 8,21 \\
\hline neutral & 16,11 & 5,17 \\
\hline weitgehend unangemessen & 21,28 & 2,74
\end{tabular}

Bei den folgenden beiden Wörtern geht es um den Vokal in der Vorsilbe $<$ Re->. Die möglichen Varianten waren die Aussprache als geschlossenes kurzes [e] (wie in See, aber kurz), als offenes kurzes $[\varepsilon]$ (wie in fett) und als reduziertes [ə] (wie beim mittleren $e$ in weitere).

\begin{tabular}{l|l|l|l} 
& \multicolumn{2}{l}{ REDAKTION } \\
\cline { 2 - 4 } & $\mathrm{R}[\mathrm{e}]$ daktion & $\mathrm{R}[\varepsilon]$ daktion & $\mathrm{R}[$ ə] daktion \\
\hline voll angemessen & 59,20 & 30,77 & 10,70 \\
\hline weitgehend angemessen & 20,07 & 23,08 & 13,38 \\
\hline neutral & 13,38 & 21,07 & 22,07 \\
\hline weitgehend unangemessen & 5,69 & 14,05 & 20,40 \\
\hline ganz unangemessen & 1,67 & 11,04 & 33,44
\end{tabular}

\begin{tabular}{l|l|l|l} 
& \multicolumn{2}{l}{ RELIGION } \\
\cline { 2 - 4 } & $\mathrm{R}[\mathrm{e}]$ ligion & $\mathrm{R}[\varepsilon]$ ligion & $\mathrm{R}[$ ə]ligion \\
\hline voll angemessen & 44,38 & 43,54 & 11,24 \\
\hline weitgehend angemessen & 21,35 & 17,98 & 14,89 \\
\hline neutral & 16,29 & 18,82 & 16,29 \\
\hline weitgehend unangemessen & 10,11 & 9,83 & 24,72 \\
\hline ganz unangemessen & 7,87 & 9,83 & 32,87
\end{tabular}


Bei Penicillin geht es um das unbetonte <e>. Die Varianten sind geschlossenes kurzes $e[\mathrm{e}]$ (wie in See, aber kurz) und offenes kurzes $e[\varepsilon]$ (wie in fett).

\begin{tabular}{|c|c|c|}
\hline & \multicolumn{2}{|c|}{ PENICILLIN } \\
\hline & $\mathrm{P}[\mathrm{e}]$ nicillin & $\mathrm{P}[\varepsilon]$ nicillin \\
\hline voll angemessen & 40,55 & 41,10 \\
\hline weitgehend angemessen & 15,24 & 18,71 \\
\hline neutral & 24,39 & 16,26 \\
\hline weitgehend unangemessen & 13,11 & 15,34 \\
\hline ganz unangemessen & 6,71 & 8,59 \\
\hline
\end{tabular}

Bei den folgenden Wörtern geht es um die Aussprache von <ei>. Die Varianten waren $e j$ [عI] oder ei [aI], bei Madeira auch noch geschlossenes langes [e:].

\begin{tabular}{l|l|l} 
& GEISHA & G[aI]sha \\
\cline { 2 - 3 } & G[EI]sha & 11,26 \\
\hline voll angemessen & 73,42 & 11,54 \\
\hline weitgehend angemessen & 18,36 & 17,86 \\
\hline neutral & 4,11 & 26,37 \\
\hline weitgehend unangemessen & 3,01 & 32,97
\end{tabular}

\begin{tabular}{|c|c|c|}
\hline & \multicolumn{2}{|c|}{ KYRIE ELEISON } \\
\hline & (Kyrie) el[عI]son & (Kyrie) el[ar]son \\
\hline voll angemessen & 71,17 & 13,77 \\
\hline weitgehend angemessen & 11,95 & 12,21 \\
\hline neutral & 12,21 & 25,97 \\
\hline weitgehend unangemessen & 2,86 & 23,90 \\
\hline ganz unangemessen & 1,82 & 24,16 \\
\hline
\end{tabular}




\begin{tabular}{l|l|l} 
& \multicolumn{2}{l}{} \\
\cline { 2 - 3 } & $\mathrm{d}\left[\mathrm{EII}_{N}\right] \mathrm{ktisch}$ & $\mathrm{d}[\mathrm{ar}] \mathrm{N}] \mathrm{ktisch}$ \\
\hline voll angemessen & 52,82 & 41,24 \\
\hline weitgehend angemessen & 13,33 & 12,37 \\
\hline neutral & 8,72 & 10,31 \\
\hline weitgehend unangemessen & 13,85 & 12,37 \\
\hline ganz unangemessen & 11,28 & 23,71
\end{tabular}

\begin{tabular}{l|l|l|l} 
& \multicolumn{2}{l}{ MADEIRA } & Mad[e:]ra \\
\cline { 2 - 4 } & Mad[EI] $] \mathrm{ra}$ & Mad[ar] $\mathrm{ra}$ & 52,05 \\
\hline voll angemessen & 33,81 & 9,40 & 21,93 \\
\hline weitgehend angemessen & 20,38 & 8,67 & 13,01 \\
\hline neutral & 17,51 & 15,18 & 6,51 \\
\hline weitgehend unangemessen & 14,87 & 21,69 & 6,51
\end{tabular}

Beim Akronym $C A D$ waren die Varianten die Aussprache als Buchstabenwort [tse:a'de:] oder wie ein Normalwort; bei letzterer war die Möglichkeit „der Schreibung entsprechend“ $a$ [kat] sowie, nach englischem Vorbild, $e$ [ket] wie in fett.

\begin{tabular}{l|l|l|l} 
& \multicolumn{2}{l}{ CAD } & [tse:a'de:] \\
\cline { 2 - 4 } & {$[\mathrm{kat}]$} & {$[\mathrm{k \varepsilon t}]$} & 52,30 \\
\hline voll angemessen & 41,14 & 12,64 & 10,92 \\
\hline weitgehend angemessen & 12,57 & 13,79 & 19,54 \\
\hline neutral & 13,71 & 17,24 & 2,30 \\
\hline weitgehend unangemessen & 10,86 & 14,37 & 14,94
\end{tabular}


Bei fahwe ging es um die Aussprache von $<$ ah $>$, wobei das $<\mathrm{h}>$ stumm sein kann, was zur Aussprache [a:] (wie in lahm) führt, oder als Frikativ realisiert werden kann, was eine Aussprache [ax] (wie in Dach) zur Folge hat.

\begin{tabular}{l|l|l} 
& JAHWE & J[ax]we \\
\cline { 2 - 3 } & J[a:]we & 43,82 \\
\hline voll angemessen & 50,56 & 12,64 \\
\hline weitgehend angemessen & 15,73 & 22,19 \\
\hline neutral & 19,10 & 9,27 \\
\hline weitgehend unangemessen & 7,58 & 12,08
\end{tabular}

\subsection{Teil 4: Lehnwörter}

Teil 4 der Umfrage widmete sich verschiedenartigen Aussprachevarianten diverser Lehnwörter aus dem Französischen und Englischen.

Bei den folgenden Wörtern französischen Ursprungs geht es um die Endung -on. Die Varianten sind -ong [эy], -on [o:n] und die nasalierte, am Französischen orientierte Aussprache [ก̃:].

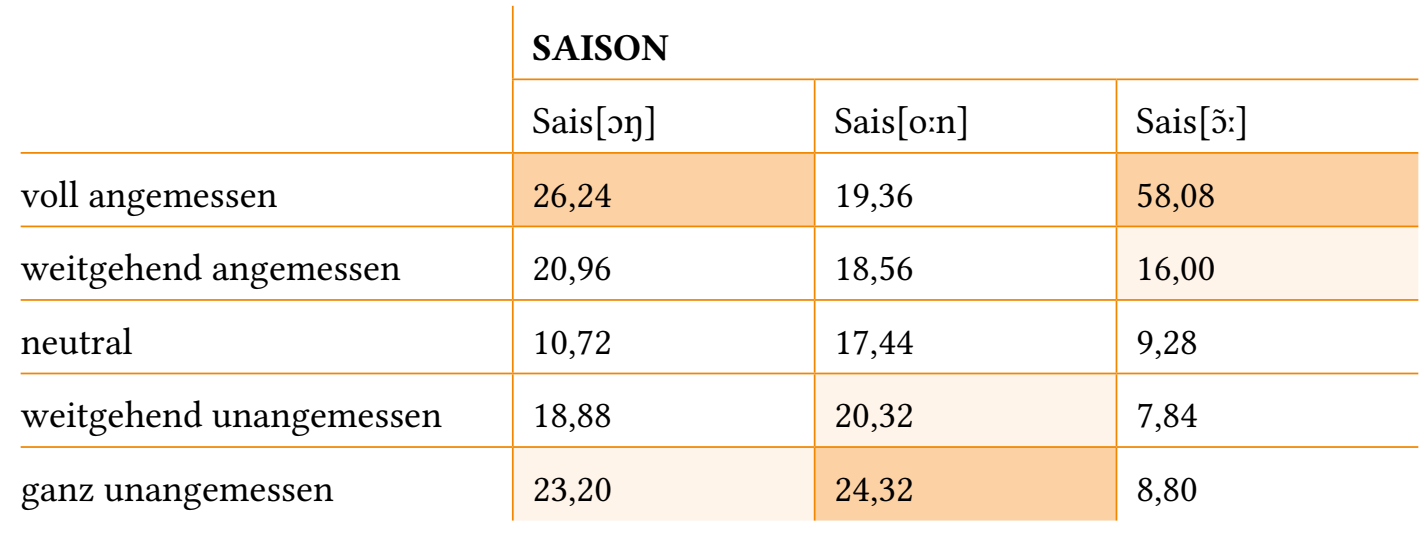

\begin{tabular}{|c|c|c|c|}
\hline & \multicolumn{3}{|l|}{ BETON } \\
\hline & $\operatorname{Bet}[$ on] & Bet[o:n] & $\operatorname{Bet}[\tilde{\jmath}:]$ \\
\hline voll angemessen & 41,86 & 29,49 & 22,88 \\
\hline weitgehend angemessen & 21,02 & 17,80 & 16,27 \\
\hline neutral & 11,19 & 13,56 & 10,85 \\
\hline weitgehend unangemessen & 11,86 & 16,78 & 22,03 \\
\hline ganz unangemessen & 14,07 & 22,37 & 27,97 \\
\hline
\end{tabular}




\begin{tabular}{l|l|l|l} 
& \multicolumn{2}{l}{ BALLON } & Ball[э̃] \\
\cline { 2 - 4 } & Ball[эy] & Ball[o:n] & 33,50 \\
\hline voll angemessen & 33,50 & 34,18 & 19,05 \\
\hline weitgehend angemessen & 19,56 & 20,07 & 11,05 \\
\hline neutral & 13,95 & 15,82 & 18,88 \\
\hline weitgehend unangemessen & 15,99 & 15,31 & 17,52
\end{tabular}

\begin{tabular}{|c|c|c|c|}
\hline & \multicolumn{3}{|c|}{ BALKON } \\
\hline & Balk[эџ] & Balk[o:n] & Balk[ñ:] \\
\hline voll angemessen & 26,10 & 60,51 & 23,05 \\
\hline weitgehend angemessen & 21,36 & 23,39 & 19,49 \\
\hline neutral & 13,73 & 7,97 & 15,93 \\
\hline weitgehend unangemessen & 17,29 & 4,41 & 20,34 \\
\hline ganz unangemessen & 21,53 & 3,73 & 21,19 \\
\hline
\end{tabular}

Bei folgenden Wörtern wurde auch nach der betonten Silbe gefragt:

\begin{tabular}{l|l|l} 
& SAISON & saiSON \\
\cline { 2 - 3 } & SAIson & 70,24 \\
\hline voll angemessen & 16,00 & 13,44 \\
\hline weitgehend angemessen & 6,88 & 4,96 \\
\hline neutral & 12,00 & 5,92 \\
\hline weitgehend unangemessen & 25,28 & 5,44
\end{tabular}

\begin{tabular}{|c|c|c|}
\hline & \multicolumn{2}{|c|}{ BETON } \\
\hline & BEton & beTON \\
\hline voll angemessen & 12,54 & 68,31 \\
\hline weitgehend angemessen & 8,31 & 17,12 \\
\hline neutral & 11,69 & 4,07 \\
\hline weitgehend unangemessen & 26,44 & 4,58 \\
\hline ganz unangemessen & 41,02 & 5,93 \\
\hline
\end{tabular}




\begin{tabular}{l|l|l} 
& BALLON & balLON \\
\cline { 2 - 3 } & BALlon & 76,06 \\
\hline voll angemessen & 9,35 & 11,71 \\
\hline weitgehend angemessen & 8,16 & 4,58 \\
\hline neutral & 8,67 & 5,43 \\
\hline weitgehend unangemessen & 28,23 & 2,21
\end{tabular}

\begin{tabular}{l|l|l} 
& BALKON & balKON \\
\cline { 2 - 3 } & BALkon & 78,31 \\
\hline voll angemessen & 9,15 & 12,20 \\
\hline weitgehend angemessen & 8,64 & 4,07 \\
\hline neutral & 8,98 & 3,56 \\
\hline weitgehend unangemessen & 27,80 & 1,86
\end{tabular}

Beim Wort Saison standen weiterhin Aussprachevarianten für < ai > zur Auswahl, und zwar: offenes kurzes $e[\varepsilon]$ und „der Schreibung entsprechend“ [ar].

\begin{tabular}{|c|c|c|}
\hline & \multicolumn{2}{|c|}{ SAISON } \\
\hline & $\mathrm{S}[\varepsilon]$ son & $\mathrm{S}[\mathrm{ar}] \mathrm{son}$ \\
\hline voll angemessen & 70,88 & 15,36 \\
\hline weitgehend angemessen & 15,68 & 8,64 \\
\hline neutral & 5,60 & 12,64 \\
\hline weitgehend unangemessen & 4,32 & 18,08 \\
\hline ganz unangemessen & 3,52 & 45,28 \\
\hline
\end{tabular}


Beim (Kassen-)Bon ging es ebenfalls um <-on>, als Varianten standen jedoch nur -ong [эy] und die am Französischen orientierte nasalierte Aussprache [̃̃̃] zur Auswahl.

\begin{tabular}{|c|c|c|}
\hline & \multicolumn{2}{|c|}{ (KASSEN-)BON } \\
\hline & $\mathrm{B}[э \mathrm{y}]$ & $\mathrm{B}[\tilde{\jmath}:]$ \\
\hline voll angemessen & 41,63 & 49,92 \\
\hline weitgehend angemessen & 19,57 & 15,59 \\
\hline neutral & 10,95 & 12,94 \\
\hline weitgehend unangemessen & 13,10 & 11,77 \\
\hline ganz unangemessen & 14,76 & 9,78 \\
\hline
\end{tabular}

Bei den folgenden Wörtern französischer Herkunft wurde die Anzahl der Silben thematisiert bzw. ob dem Buchstaben <e> am Ende bzw. in der Mitte der folgenden Wörter ein vokalischer Lautwert zukommt (Promille usw.) oder ob er stumm bleibt (d.h. „Promill“ usw. gesprochen wird).

\begin{tabular}{|c|c|c|}
\hline & \multicolumn{2}{|c|}{ PROMILLE } \\
\hline & Promille & Promill \\
\hline voll angemessen & 69,04 & 23,29 \\
\hline weitgehend angemessen & 15,34 & 16,44 \\
\hline neutral & 9,04 & 20,82 \\
\hline weitgehend unangemessen & 4,38 & 20,82 \\
\hline ganz unangemessen & 2,19 & 18,63 \\
\hline
\end{tabular}

\begin{tabular}{l|l|l} 
& \multicolumn{2}{l}{ OMELETT } \\
\cline { 2 - 3 } & Omelett & Omlett \\
\hline voll angemessen & 3,94 & 86,06 \\
\hline weitgehend angemessen & 4,24 & 8,79 \\
\hline neutral & 7,27 & 2,12 \\
\hline weitgehend unangemessen & 25,76 & 1,21 \\
\hline ganz unangemessen & 58,79 & 1,82
\end{tabular}




\begin{tabular}{l|l|l} 
& KOTELETT & Kotlett \\
\cline { 2 - 3 } & Kotelett & 86,52 \\
\hline voll angemessen & 2,51 & 9,72 \\
\hline weitgehend angemessen & 3,76 & 2,51 \\
\hline neutral & 9,09 & 0,00 \\
\hline weitgehend unangemessen & 30,09 & 1,25 \\
\hline ganz unangemessen & 54,55 & \\
\hline voll angemessen & KOTELETTEN & Kotletten \\
\hline weitgehend angemessen & Koteletten & 70,75 \\
\hline neutral & 11,95 & 14,15 \\
\hline weitgehend unangemessen & 7,23 & 6,29 \\
\hline ganz unangemessen & 11,95 & 4,09 \\
\hline
\end{tabular}

Bei Omelett und Kotelett wurde auch nach der betonten Silbe gefragt.

\begin{tabular}{|c|c|c|}
\hline & \multicolumn{2}{|c|}{ OMELETT } \\
\hline & OM(e)lett & om(e)LETT \\
\hline voll angemessen & 28,48 & 60,00 \\
\hline weitgehend angemessen & 19,09 & 16,36 \\
\hline neutral & 18,48 & 9,70 \\
\hline weitgehend unangemessen & 18,79 & 9,70 \\
\hline ganz unangemessen & 15,15 & 4,24 \\
\hline
\end{tabular}

\begin{tabular}{l|l|l} 
& \multicolumn{2}{l}{ KOTELETT } \\
\cline { 2 - 3 } & KOt(e)lett & kot(e)LETT \\
\hline voll angemessen & 35,11 & 50,78 \\
\hline weitgehend angemessen & 13,79 & 17,24 \\
\hline neutral & 9,40 & 11,60 \\
\hline weitgehend unangemessen & 18,81 & 10,97 \\
\hline ganz unangemessen & 22,88 & 9,40
\end{tabular}


Bei Ressourcen geht es um die Aussprache von <ou>. Die Varianten waren $u$ [ $\mho$ ] (offen und kurz, wie in Butter) und o [0] (offen und kurz, wie in hoffen).

\begin{tabular}{|c|c|c|}
\hline & \multicolumn{2}{|c|}{ RESSOURCEN } \\
\hline & Ress[v]rcen & Ress[o]rcen \\
\hline voll angemessen & 75,68 & 12,50 \\
\hline weitgehend angemessen & 14,29 & 12,20 \\
\hline neutral & 3,95 & 11,59 \\
\hline weitgehend unangemessen & 2,43 & 26,83 \\
\hline ganz unangemessen & 3,65 & 36,89 \\
\hline
\end{tabular}

Bei Curry ging es um die Aussprache von $<\mathrm{u}>$. Die Varianten sind $\ddot{o}$ [œ] (offen und kurz wie in Löffel) und (kurzes) $a$ [a] (wie in Lamm). Leider wurde bei der Konzeption der Umfrage übersehen, auch nach der Variante $e$ zu fragen, die im Osten Deutschlands gebräuchlich ist.

\begin{tabular}{l|l|l} 
& CURRY & \multicolumn{2}{l}{} \\
\cline { 2 - 3 } & C[œ]rry & C $]$ ]ry \\
\hline voll angemessen & 82,64 & 4,97 \\
\hline weitgehend angemessen & 11,29 & 3,31 \\
\hline neutral & 1,93 & 6,91 \\
\hline weitgehend unangemessen & 2,48 & 20,17 \\
\hline ganz unangemessen & 1,65 & 64,64
\end{tabular}

Die folgenden Wörter beginnen alle mit $<\mathrm{J}->$. Die zu beurteilenden Varianten waren: $s c h$ [S] sowie dessen stimmhaftes Gegenstück [3] und als Alternative die Affrikate $d s c h$ [dz].

\begin{tabular}{l|l|l|l} 
& \multicolumn{2}{l}{ JOURNALIST } \\
\cline { 2 - 4 } & [J]ournalist & [3]ournalist & [d]]ournalist \\
\hline voll angemessen & 31,15 & 68,85 & 3,72 \\
\hline weitgehend angemessen & 15,93 & 9,38 & 6,56 \\
\hline neutral & 11,50 & 6,90 & 9,04 \\
\hline weitgehend unangemessen & 15,58 & 6,37 & 21,28 \\
\hline ganz unangemessen & 25,84 & 8,50 & 59,40
\end{tabular}




\begin{tabular}{|c|c|c|c|}
\hline & \multicolumn{3}{|c|}{ JAMAIKA } \\
\hline & [S]amaika & [3]amaika & [dz]amaika \\
\hline voll angemessen & 3,81 & 46,85 & 39,17 \\
\hline weitgehend angemessen & 5,30 & 12,91 & 14,88 \\
\hline neutral & 9,77 & 12,25 & 9,59 \\
\hline weitgehend unangemessen & 27,98 & 10,43 & 15,54 \\
\hline \multirow[t]{3}{*}{ ganz unangemessen } & 53,15 & 17,55 & 20,83 \\
\hline & \multicolumn{3}{|l|}{ JURY } \\
\hline & [J]ury & [3]ury & {$\left[\mathrm{d}_{3}\right]$ ury } \\
\hline voll angemessen & 24,29 & 59,46 & 17,92 \\
\hline weitgehend angemessen & 13,40 & 11,73 & 15,24 \\
\hline neutral & 13,23 & 8,71 & 11,56 \\
\hline weitgehend unangemessen & 18,59 & 7,87 & 23,12 \\
\hline ganz unangemessen & 30,49 & 12,23 & 32,16 \\
\hline
\end{tabular}

Bei fury wurde zudem nach der betonten Silbe gefragt:

\begin{tabular}{l|l|l} 
& JURY & juRY \\
\cline { 2 - 3 } & JUry & 23,41 \\
\hline voll angemessen & 71,40 & 14,55 \\
\hline weitgehend angemessen & 14,72 & 15,05 \\
\hline neutral & 8,70 & 25,75 \\
\hline weitgehend unangemessen & 3,18 & 21,24
\end{tabular}

Bei den beiden nächsten Wörtern war ebenfalls die Aussprache von $<\mathrm{J}->$ Thema. Die Varianten sind hier $d s c h$ [d3] und „der Schreibung entsprechend“ [j].

\begin{tabular}{l|l|l} 
& \multicolumn{2}{|l}{ JAVA (Programmiersprache) } \\
\cline { 2 - 3 } & {$[$ d3]ava } & {$[\mathrm{j}]$ ava } \\
\hline voll angemessen & 31,07 & 63,97 \\
\hline weitgehend angemessen & 11,49 & 16,19 \\
\hline neutral & 11,75 & 8,62 \\
\hline weitgehend unangemessen & 14,88 & 6,79 \\
\hline ganz unangemessen & 30,81 & 4,44
\end{tabular}




\begin{tabular}{l|l|l} 
& \multicolumn{2}{l}{ JUMBO (Flugzeugtyp) } \\
\cline { 2 - 3 } & {$[\mathrm{d} 3]$ umbo } & {$[\mathrm{j}] \mathrm{umbo}$} \\
\hline voll angemessen & 76,21 & 15,61 \\
\hline weitgehend angemessen & 13,11 & 12,93 \\
\hline neutral & 4,13 & 15,85 \\
\hline weitgehend unangemessen & 2,91 & 28,05 \\
\hline ganz unangemessen & 3,64 & 27,56
\end{tabular}

Die folgenden aus dem Englischen stammenden Wörter enden alle auf $<-$ ey $>$. Die abgefragten Varianten waren - $e j$ [EI], [e] (wie in Frottee) und [i] (wie in Lolli).

\begin{tabular}{l|l|l|l} 
& \multicolumn{2}{|l}{ HOCKEY } & Hock[i] \\
\cline { 2 - 4 } & Hock[EI] & Hock[e] & 28,01 \\
\hline voll angemessen & 58,38 & 19,90 & 17,80 \\
\hline weitgehend angemessen & 22,25 & 20,68 & 10,21 \\
\hline neutral & 9,95 & 15,71 & 22,51 \\
\hline weitgehend unangemessen & 6,28 & 21,73 & 21,47
\end{tabular}

\begin{tabular}{|c|c|c|c|}
\hline & \multicolumn{3}{|c|}{ TROLLEY } \\
\hline & $\operatorname{Troll}[\varepsilon \mathrm{I}]$ & Troll[e] & Troll[i] \\
\hline voll angemessen & 30,08 & 6,77 & 56,00 \\
\hline weitgehend angemessen & 22,31 & 12,78 & 20,75 \\
\hline neutral & 13,78 & 12,28 & 8,50 \\
\hline weitgehend unangemessen & 17,29 & 29,32 & 7,75 \\
\hline ganz unangemessen & 16,54 & 38,85 & 7,00 \\
\hline
\end{tabular}

\begin{tabular}{l|l|l|l} 
& \multicolumn{2}{l}{ CHUTNEY } \\
\cline { 2 - 4 } & Chutn[عI] & Chutn[e] & Chutn[i] \\
\hline voll angemessen & 62,76 & 13,02 & 28,39 \\
\hline weitgehend angemessen & 21,09 & 22,14 & 17,45 \\
\hline neutral & 6,77 & 19,01 & 15,89 \\
\hline weitgehend unangemessen & 4,69 & 24,22 & 16,15 \\
\hline ganz unangemessen & 4,69 & 21,61 & 22,14
\end{tabular}




\begin{tabular}{|c|c|c|c|}
\hline & \multicolumn{3}{|c|}{ VOLLEY(BALL) } \\
\hline & $\operatorname{Voll}[\varepsilon I]$ & Voll[e] & Voll[i] \\
\hline voll angemessen & 49,86 & 24,11 & 22,74 \\
\hline weitgehend angemessen & 22,74 & 26,03 & 17,26 \\
\hline neutral & 13,15 & 21,92 & 12,33 \\
\hline weitgehend unangemessen & 7,40 & 14,25 & 20,55 \\
\hline ganz unangemessen & 6,85 & 13,70 & 27,12 \\
\hline
\end{tabular}

\begin{tabular}{l|l|l|l} 
& \multicolumn{2}{l}{ WEMBLEY } & \multicolumn{2}{l}{} \\
\cline { 2 - 4 } & Wembl[ $[\mathrm{r}]$ & Wembl[e] & Wembl[i] \\
\hline voll angemessen & 59,32 & 6,84 & 24,21 \\
\hline weitgehend angemessen & 23,88 & 19,74 & 18,68 \\
\hline neutral & 8,66 & 18,42 & 14,47 \\
\hline weitgehend unangemessen & 3,67 & 26,32 & 17,63 \\
\hline ganz unangemessen & 4,46 & 28,68 & 25,00
\end{tabular}

\begin{tabular}{l|l|l|l} 
& \multicolumn{2}{|l}{ WRIGLEY } & Wrigl[i] \\
\cline { 2 - 4 } & Wrigl[घI] & Wrigl[e] & 29,63 \\
\hline voll angemessen & 63,27 & 9,26 & 13,58 \\
\hline weitgehend angemessen & 14,51 & 12,96 & 12,04 \\
\hline neutral & 8,64 & 17,28 & 18,52 \\
\hline weitgehend unangemessen & 6,48 & 27,47 & 26,23
\end{tabular}

Bei Trainer und Training geht es um die Aussprache von $<$ ai $>$. Die Varianten waren $-e j[\varepsilon I]$, geschlossenes langes [e:] (wie in See) und offenes langes [ع:] (wie in fett, aber lang).

\begin{tabular}{l|l|l|l} 
& \multicolumn{2}{l}{ TRAINER } & $\operatorname{Tr}[\varepsilon:] n e r$ \\
\cline { 2 - 4 } & $\operatorname{Tr}[\varepsilon \mathrm{r}] \mathrm{n}$ er & $\operatorname{Tr}[\mathrm{e}:] \mathrm{ner}$ & 27,23 \\
\hline voll angemessen & 47,77 & 31,68 & 21,29 \\
\hline weitgehend angemessen & 16,58 & 25,00 & 15,59 \\
\hline neutral & 13,86 & 17,82 & 22,28 \\
\hline weitgehend unangemessen & 13,12 & 14,60 & 13,61
\end{tabular}




\begin{tabular}{l|l|l|l} 
& \multicolumn{2}{l}{ TRAINING } \\
\cline { 2 - 4 } & $\operatorname{Tr}[\varepsilon \mathrm{r}]$ ning & $\operatorname{Tr}[\mathrm{e}:]$ ning & $\operatorname{Tr}[\varepsilon:]$ ning \\
\hline voll angemessen & 45,19 & 36,39 & 28,29 \\
\hline weitgehend angemessen & 17,28 & 25,00 & 23,08 \\
\hline neutral & 15,80 & 16,83 & 14,64 \\
\hline weitgehend unangemessen & 12,59 & 11,39 & 20,10 \\
\hline ganz unangemessen & 9,14 & 10,40 & 13,90
\end{tabular}

Bei Puzzle und bluffen geht es um die Aussprache von $<\mathrm{u}>$. Die Varianten waren (kurzes) $a$ [a], „der Schreibung entsprechend" [ซ] (offen und kurz wie in Butter) und bei bluffen auch kurzes, offenes $\ddot{o}[œ]$.

\begin{tabular}{|c|c|c|}
\hline & \multicolumn{2}{|l|}{ PUZZLE } \\
\hline & $\mathrm{P}[\mathrm{a}]$ zzle & $\mathrm{P}[\mho]$ zzle \\
\hline voll angemessen & 37,46 & 63,13 \\
\hline weitgehend angemessen & 17,70 & 17,99 \\
\hline neutral & 16,22 & 7,67 \\
\hline weitgehend unangemessen & 15,63 & 7,37 \\
\hline ganz unangemessen & 12,98 & 3,83 \\
\hline
\end{tabular}

\begin{tabular}{l|l|l|l} 
& \multicolumn{2}{|l}{ BLUFFEN } \\
\cline { 2 - 4 } & bl[œ]ffen & bl[a]ffen & bl[ช]ffen \\
\hline voll angemessen & 75,38 & 13,94 & 5,18 \\
\hline weitgehend angemessen & 15,81 & 8,48 & 4,27 \\
\hline neutral & 3,65 & 12,42 & 7,32 \\
\hline weitgehend unangemessen & 2,74 & 23,64 & 16,77 \\
\hline ganz unangemessen & 2,43 & 41,52 & 66,46
\end{tabular}


Bei den folgenden acht Wörtern englischen Ursprungs war die Aussprache von $<\mathrm{a}>$ Thema. Die Varianten sind offenes kurzes $e$ (wie in fett) $[\varepsilon]$, offenes langes [ $\varepsilon$ :] (wie in fett, aber lang) und zum Teil auch $a$ [a:].

\begin{tabular}{l|l|l} 
& ADMIN & {$[\varepsilon]$ dmin } \\
\cline { 2 - 3 } & {$[$ a]dmin } & 33,33 \\
\hline voll angemessen & 64,77 & 12,47 \\
\hline weitgehend angemessen & 17,89 & 16,26 \\
\hline neutral & 8,40 & 18,97 \\
\hline weitgehend unangemessen & 6,23 & 18,97
\end{tabular}

\begin{tabular}{|c|c|c|}
\hline & \multicolumn{2}{|l|}{ AIRBAG } \\
\hline & $\operatorname{Airb}[\varepsilon] \mathrm{g}$ & $\operatorname{Airb}[\varepsilon:] g$ \\
\hline voll angemessen & 54,19 & 33,25 \\
\hline weitgehend angemessen & 19,11 & 14,66 \\
\hline neutral & 12,04 & 16,49 \\
\hline weitgehend unangemessen & 10,21 & 19,11 \\
\hline ganz unangemessen & 4,45 & 16,49 \\
\hline
\end{tabular}

\begin{tabular}{|c|c|c|}
\hline & \multicolumn{2}{|l|}{ GAG } \\
\hline & $\mathrm{G}[\varepsilon] \mathrm{g}$ & $\mathrm{G}[\varepsilon:] \mathrm{g}$ \\
\hline voll angemessen & 59,84 & 30,87 \\
\hline weitgehend angemessen & 18,31 & 15,30 \\
\hline neutral & 8,74 & 13,93 \\
\hline weitgehend unangemessen & 10,11 & 24,04 \\
\hline ganz unangemessen & 3,01 & 15,85 \\
\hline
\end{tabular}

\begin{tabular}{l|l|l} 
& \multicolumn{2}{|l}{ MONTANA (US-Bundesstaat) } \\
\cline { 2 - 3 } & Mont[a:]na & Mont[ع:]na \\
\hline voll angemessen & 35,94 & 52,60 \\
\hline weitgehend angemessen & 20,05 & 21,61 \\
\hline neutral & 16,41 & 10,16 \\
\hline weitgehend unangemessen & 16,41 & 7,81 \\
\hline ganz unangemessen & 11,20 & 7,81
\end{tabular}




\begin{tabular}{l|l|l|l} 
& \multicolumn{2}{l}{ RAFTING } & \multicolumn{2}{l}{} \\
\cline { 2 - 4 } & $\mathrm{R}[\varepsilon] \mathrm{fting}$ & $\mathrm{R}[\varepsilon:] \mathrm{fting}$ & $\mathrm{R}[\mathrm{a}:] \mathrm{fting}$ \\
\hline voll angemessen & 23,64 & 19,81 & 59,29 \\
\hline weitgehend angemessen & 15,37 & 16,75 & 12,94 \\
\hline neutral & 15,37 & 16,27 & 10,12 \\
\hline weitgehend unangemessen & 23,17 & 22,88 & 8,94 \\
\hline ganz unangemessen & 22,46 & 24,29 & 8,71
\end{tabular}

\begin{tabular}{|c|c|c|c|}
\hline & \multicolumn{3}{|l|}{ SAMPLE } \\
\hline & $\mathrm{S}[\varepsilon] \mathrm{mple}$ & $\mathrm{S}[\varepsilon:] \mathrm{mple}$ & S[a:]mple \\
\hline voll angemessen & 41,83 & 36,47 & 22,86 \\
\hline weitgehend angemessen & 20,63 & 17,38 & 10,57 \\
\hline neutral & 12,89 & 14,53 & 11,43 \\
\hline weitgehend unangemessen & 14,04 & 18,80 & 22,29 \\
\hline ganz unangemessen & 10,60 & 12,82 & 32,86 \\
\hline
\end{tabular}

\begin{tabular}{|c|c|c|}
\hline & \multicolumn{2}{|l|}{ TABSTOPP } \\
\hline & $\mathrm{T}[\mathrm{a}]$ bstopp & $\mathrm{T}[\varepsilon]$ bstopp \\
\hline voll angemessen & 43,58 & 43,73 \\
\hline weitgehend angemessen & 17,65 & 12,53 \\
\hline neutral & 14,17 & 11,20 \\
\hline weitgehend unangemessen & 14,97 & 14,93 \\
\hline ganz unangemessen & 9,63 & 17,60 \\
\hline
\end{tabular}

\begin{tabular}{l|l|l} 
& ZAPPEN & $z[\varepsilon]$ ppen \\
\cline { 2 - 3 } & z[a]ppen & 60,95 \\
\hline voll angemessen & 26,12 & 16,89 \\
\hline weitgehend angemessen & 10,55 & 6,86 \\
\hline neutral & 12,93 & 5,80 \\
\hline weitgehend unangemessen & 25,33 & 9,50
\end{tabular}


Auch bei Upgrade war die Aussprache von <a $>$ zu beurteilen, die Varianten waren hier $e j$ [EI] und geschlossenes langes $e[\mathrm{e}:]$.

\begin{tabular}{l|l|l} 
& UPGRADE & Upgr[e:]de \\
\cline { 2 - 3 } & Upgr[EI]de & 3,97 \\
\hline voll angemessen & 79,16 & 5,46 \\
\hline weitgehend angemessen & 14,64 & 14,14 \\
\hline neutral & 3,47 & 39,21 \\
\hline weitgehend unangemessen & 1,24 & 37,22
\end{tabular}

Weiterhin wurde bei Upgrade nach der betonten Silbe gefragt:

\begin{tabular}{l|l|l} 
& \multicolumn{2}{l}{ UPGRADE } \\
\cline { 2 - 3 } & UPgrade & upGRADE \\
\hline voll angemessen & 86,85 & 4,22 \\
\hline weitgehend angemessen & 9,18 & 5,21 \\
\hline neutral & 2,23 & 10,92 \\
\hline weitgehend unangemessen & 1,24 & 35,98 \\
\hline ganz unangemessen & 0,50 & 43,67
\end{tabular}

Bei Cinch(-Stecker) geht es um die Aussprache des initialen $<\mathrm{C}->$. Die Varianten waren $t s c h[\mathrm{t} f], t s[\mathrm{ts}], s[\mathrm{~s}]$ und $k[\mathrm{k}]$.

\begin{tabular}{l|l|l|l|l} 
& \multicolumn{4}{l}{ CINCH(-STECKER) } \\
\cline { 2 - 5 } & {$[\mathrm{t}]$ ]inch } & {$[\mathrm{ts}]$ inch } & {$[\mathrm{s}]$ inch } & {$[\mathrm{k}]$ inch } \\
\hline voll angemessen & 70,39 & 5,03 & 8,94 & 5,06 \\
\hline weitgehend angemessen & 11,73 & 5,59 & 3,35 & 5,62 \\
\hline neutral & 5,59 & 8,94 & 10,06 & 10,11 \\
\hline weitgehend unangemessen & 2,79 & 21,79 & 13,97 & 11,80 \\
\hline ganz unangemessen & 9,50 & 58,66 & 63,69 & 67,42
\end{tabular}


Bei den folgenden beiden Wörtern geht es um die Aussprache von $<\mathrm{Z} / \mathrm{z}>$. Die möglichen Varianten waren $t s[\mathrm{ts}]$ und $s[\mathrm{z}]$.

\begin{tabular}{l|l|l} 
& ZIP(-DATEI) & {$[\mathrm{z}] \mathrm{ip}$} \\
\cline { 2 - 3 } & {$[$ ts]ip } & 43,75 \\
\hline voll angemessen & 47,92 & 11,90 \\
\hline weitgehend angemessen & 18,75 & 8,63 \\
\hline neutral & 8,63 & 16,07 \\
\hline weitgehend unangemessen & 13,39 & 19,64
\end{tabular}

\begin{tabular}{|c|c|c|}
\hline & \multicolumn{2}{|l|}{ GEZIPPT } \\
\hline & ge[ts]ippt & ge[z]ippt \\
\hline voll angemessen & 46,73 & 38,39 \\
\hline weitgehend angemessen & 19,05 & 10,12 \\
\hline neutral & 9,23 & 9,82 \\
\hline weitgehend unangemessen & 13,39 & 17,26 \\
\hline ganz unangemessen & 11,61 & 24,40 \\
\hline
\end{tabular}

In Roadmap und Toast geht es um den Vokal hinter der Schreibung < oa>. Die Varianten waren hier geschlossenes langes $o$ [o:] (wie in Hof) sowie der im Deutschen in heimischen Wörtern nicht vorhandene Diphthong ou [ơ్ ].

\begin{tabular}{|c|c|c|}
\hline & \multicolumn{2}{|c|}{ ROADMAP } \\
\hline & $\mathrm{R}[\mathrm{o:}] \mathrm{dmap}$ & $\mathrm{R}[\mathrm{s}$ ] $] \mathrm{dmap}$ \\
\hline voll angemessen & 9,32 & 76,16 \\
\hline weitgehend angemessen & 10,14 & 12,88 \\
\hline neutral & 17,26 & 4,38 \\
\hline weitgehend unangemessen & 34,25 & 5,48 \\
\hline ganz unangemessen & 29,04 & 1,10 \\
\hline
\end{tabular}




\begin{tabular}{|c|c|c|}
\hline & \multicolumn{2}{|c|}{ TOAST (Brot) } \\
\hline & $\mathrm{T}[\mathrm{o}:] \mathrm{st}$ & $\mathrm{T}[\mathrm{oz}] \mathrm{N}$ st \\
\hline voll angemessen & 63,45 & 35,51 \\
\hline weitgehend angemessen & 21,15 & 16,97 \\
\hline neutral & 9,92 & 13,58 \\
\hline weitgehend unangemessen & 3,13 & 17,49 \\
\hline \multirow[t]{3}{*}{ ganz unangemessen } & 2,35 & 16,45 \\
\hline & \multicolumn{2}{|c|}{ TOAST (Trinkspruch) } \\
\hline & $\mathrm{T}[\mathrm{o}:] \mathrm{st}$ & $\mathrm{T}[\mathrm{oz}] \mathrm{N} \mathrm{st}$ \\
\hline voll angemessen & 42,30 & 44,39 \\
\hline weitgehend angemessen & 18,54 & 15,40 \\
\hline neutral & 15,14 & 12,53 \\
\hline weitgehend unangemessen & 14,10 & 13,32 \\
\hline ganz unangemessen & 9,92 & 14,36 \\
\hline
\end{tabular}

Bei Shampoo geht es zunächst um die betonte Silbe.

\begin{tabular}{|c|c|c|}
\hline & \multicolumn{2}{|l|}{ SHAMPOO } \\
\hline & SHAMpoo & shamPOO \\
\hline voll angemessen & 57,78 & 25,08 \\
\hline weitgehend angemessen & 16,83 & 19,68 \\
\hline neutral & 9,52 & 18,10 \\
\hline weitgehend unangemessen & 12,38 & 18,73 \\
\hline ganz unangemessen & 3,49 & 18,41 \\
\hline
\end{tabular}


Darüber hinaus geht es bei diesem Wort um die Aussprache von <0o>. Die Varianten waren die Aussprache als $o$ [o] (wie in Kino), on [on] (mit kurzem offenem o wie in hoffen), langem $u$ [u:] (wie in $H u t$ ) oder kürzerem $u[\mathrm{u}]$.

\begin{tabular}{l|l|l|l|l} 
& \multicolumn{4}{l}{ SHAMPOO } \\
\cline { 2 - 5 } & Shamp[o] & Shamp[on] & Shamp[u:] & Shamp[u] \\
\hline voll angemessen & 24,13 & 2,86 & 41,90 & 22,86 \\
\hline weitgehend angemessen & 14,92 & 3,49 & 22,86 & 21,27 \\
\hline neutral & 14,60 & 6,03 & 12,06 & 15,56 \\
\hline weitgehend unangemessen & 18,41 & 22,22 & 8,89 & 17,46 \\
\hline ganz unangemessen & 27,94 & 65,40 & 14,29 & 22,86
\end{tabular}

Die folgenden Wörter beginnen mit $<$ S $>$. Die Aussprachevarianten waren hier stimmloses [s] vs. stimmhaftes [z].

\begin{tabular}{l|l|l} 
& SEX & {$[\mathrm{z}] \mathrm{ex}$} \\
\cline { 2 - 3 } & {$[\mathrm{s}] \mathrm{ex}$} & 20,15 \\
\hline voll angemessen & 75,50 & 5,47 \\
\hline weitgehend angemessen & 10,15 & 8,96 \\
\hline neutral & 4,70 & 23,38 \\
\hline weitgehend unangemessen & 4,70 & 42,04
\end{tabular}

\begin{tabular}{|c|c|c|}
\hline & \multicolumn{2}{|l|}{ SINGLE } \\
\hline & {$[\mathrm{s}]$ ingle } & [z]ingle \\
\hline voll angemessen & 69,50 & 18,25 \\
\hline weitgehend angemessen & 9,50 & 10,75 \\
\hline neutral & 7,25 & 12,75 \\
\hline weitgehend unangemessen & 7,25 & 22,00 \\
\hline ganz unangemessen & 6,50 & 36,25 \\
\hline
\end{tabular}




\begin{tabular}{l|l|l} 
& SONG & {$[\mathrm{z}]$ ong } \\
\cline { 2 - 3 } & {$[\mathrm{s}]$ ong } & 34,13 \\
\hline voll angemessen & 55,15 & 10,85 \\
\hline weitgehend angemessen & 10,03 & 10,05 \\
\hline neutral & 9,50 & 16,93 \\
\hline weitgehend unangemessen & 13,46 & 28,04
\end{tabular}

Bei den folgenden Wörtern geht es um das $<\mathrm{z}>$ am Wortanfang. Die Varianten waren $t s$ [ts] und $s$. Das $s$ kann dabei, abhängig von den Sprechgewohnheiten, neben stimmhaftem [z] auch stimmloses [s] sein.

\begin{tabular}{l|l|l} 
& ZAPPEN & [ts]appen \\
\cline { 2 - 3 } & [z]appen & 43,80 \\
\hline voll angemessen & 44,62 & 13,72 \\
\hline weitgehend angemessen & 13,39 & 11,61 \\
\hline neutral & 10,76 & 17,94 \\
\hline weitgehend unangemessen & 14,96 & 12,93
\end{tabular}

\begin{tabular}{l|l|l} 
& ZOOMEN & {$[$ ts]oomen } \\
\cline { 2 - 3 } & {$[\mathrm{z}]$ oomen } & 19,74 \\
\hline voll angemessen & 72,28 & 10,91 \\
\hline weitgehend angemessen & 12,69 & 7,01 \\
\hline neutral & 4,66 & 27,79 \\
\hline weitgehend unangemessen & 4,15 & 34,55
\end{tabular}


Die folgenden englischen Wörter beginnen alle mit bzw. enthalten $\langle\mathrm{W} / \mathrm{w}\rangle$. Die Varianten waren hier die eingedeutschte Aussprache mit [v] wie in Wasser oder die englische Originalaussprache mit $[\mathrm{w}]$.

\begin{tabular}{|c|c|c|}
\hline & \multicolumn{2}{|c|}{ WASHINGTON } \\
\hline & [v]ashington & [w] ashington \\
\hline voll angemessen & 20,10 & 80,93 \\
\hline weitgehend angemessen & 14,95 & 10,31 \\
\hline neutral & 15,98 & 7,22 \\
\hline weitgehend unangemessen & 26,29 & 1,03 \\
\hline ganz unangemessen & 22,68 & 0,52 \\
\hline
\end{tabular}

\begin{tabular}{l|l|l} 
& HAWAII & Ha[w]aii \\
\cline { 2 - 3 } & Ha[v]aii & 50,75 \\
\hline voll angemessen & 61,85 & 17,75 \\
\hline weitgehend angemessen & 19,20 & 18,25 \\
\hline neutral & 11,72 & 8,25 \\
\hline weitgehend unangemessen & 4,74 & 5,00
\end{tabular}

\begin{tabular}{l|l|l} 
& WEB & {$[$ w]eb } \\
\cline { 2 - 3 } & {$[\mathrm{v}] \mathrm{eb}$} & 66,13 \\
\hline voll angemessen & 47,03 & 12,37 \\
\hline weitgehend angemessen & 18,65 & 10,22 \\
\hline neutral & 15,14 & 7,26 \\
\hline weitgehend unangemessen & 12,70 & 4,03
\end{tabular}


Bei Lounge und Relaunch sind, trotz unterschiedlicher Schreibung, die gleichen Aussprachevarianten für die betonten Vokale vorhanden. Die Varianten waren au [aṽ ] und offenes langes $o[\mathrm{~s}]$ (wie in hoffen, aber lang).

\begin{tabular}{l|l|l} 
& LOUNGE & L[э:]nge \\
\cline { 2 - 3 } & L[aro]nge & 31,54 \\
\hline voll angemessen & 55,26 & 17,36 \\
\hline weitgehend angemessen & 18,34 & 16,14 \\
\hline neutral & 11,74 & 17,85 \\
\hline weitgehend unangemessen & 9,05 & 17,11
\end{tabular}

\begin{tabular}{|c|c|c|}
\hline & \multicolumn{2}{|c|}{ RELAUNCH } \\
\hline & Rel[aş]nch & $\operatorname{Rel}[э:] \mathrm{nch}$ \\
\hline voll angemessen & 28,91 & 56,93 \\
\hline weitgehend angemessen & 17,99 & 10,62 \\
\hline neutral & 12,39 & 10,62 \\
\hline weitgehend unangemessen & 19,76 & 15,93 \\
\hline ganz unangemessen & 20,94 & 5,90 \\
\hline
\end{tabular}

Bei Nuance geht es einerseits um die Aussprache von „an“, hier konkurriert, wie in den auf -on endenden Fällen, -ang [aך], mit der am Französischen orientierten nasalierten Aussprache [ã]. Weiterhin ging es darum, ob das $<\mathrm{e}>$ am Ende mitgesprochen wird oder stumm bleibt.

\begin{tabular}{|c|c|c|c|c|}
\hline & \multicolumn{4}{|l|}{ NUANCE } \\
\hline & $\mathrm{Nu}$ [aysə] & $\mathrm{Nu}[\mathrm{ays}]$ & $\mathrm{Nu}[\tilde{a}: s ə]$ & $\mathrm{Nu}[\tilde{a}: s]$ \\
\hline voll angemessen & 6,77 & 7,59 & 40,26 & 49,67 \\
\hline weitgehend angemessen & 9,24 & 9,41 & 22,11 & 17,49 \\
\hline neutral & 10,23 & 9,57 & 11,22 & 11,55 \\
\hline weitgehend unangemessen & 24,26 & 27,72 & 13,37 & 12,05 \\
\hline ganz unangemessen & 49,50 & 45,71 & 13,04 & 9,24 \\
\hline
\end{tabular}


Zudem wurden bei diesem Wort die Aussprachevarianten von $<\mathrm{u}>$ abgefragt. Die Varianten waren „der Schreibung entsprechend“, also geschlossenes kurzes $u[u]$ (wie in Hut, aber kurz) sowie geschlossenes kurzes $\ddot{u}[\mathrm{y}]$ (wie in Lüge, aber kurz).

\begin{tabular}{l|l|l} 
& NUANCE & N[y]ance \\
\cline { 2 - 3 } & N[u]ance & 35,48 \\
\hline voll angemessen & 61,06 & 12,05 \\
\hline weitgehend angemessen & 13,53 & 14,85 \\
\hline neutral & 6,44 & 17,16 \\
\hline weitgehend unangemessen & 7,59 & 20,46
\end{tabular}

Bei Emblem geht es um die Aussprache von Em- (erste Silbe). Die Varianten sind „der Schreibung entsprechend", also em- [ $\mathrm{\varepsilon m}$ ], die nasalierte, am Französischen orientierte Aussprache [ã], bei der das folgende $m$ nicht gesprochen wird, sowie eine nasalierte Aussprache bei mitgesprochenem folgendem $m$ [ãm].

\begin{tabular}{l|l|l|l} 
& \multicolumn{2}{l}{ EMBLEM } & {$[\tilde{a}$ ] blem } \\
\cline { 2 - 4 } & {$[\varepsilon \mathrm{cm}] \mathrm{blem}$} & {$[\tilde{a}] \mathrm{blem}$} & 11,88 \\
\hline voll angemessen & 68,65 & 11,40 & 15,89 \\
\hline weitgehend angemessen & 15,76 & 10,75 & 20,39 \\
\hline neutral & 7,07 & 19,90 & 23,76 \\
\hline weitgehend unangemessen & 4,34 & 26,65 & 28,09
\end{tabular}

Hier konkurrieren die am Französischen orientierte Aussprache [mi'flẽ:] und die eingedeutschte Variante [miçə'li:n]. Leider wurde bei der Abfrage keine Bedeutung spezifiziert, so dass bei den Antworten sowohl der Reifenhersteller als auch der - vom selben Unternehmen herausgegebene - Restaurantführer gemeint sein kann (was für die Wahl der angemessenen Aussprachevariante eine erhebliche Rolle spielen dürfte).

\begin{tabular}{|c|c|c|}
\hline & \multicolumn{2}{|c|}{ MICHELIN } \\
\hline & 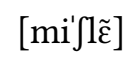 & [miçə'li:n] \\
\hline voll angemessen & 52,37 & 43,22 \\
\hline weitgehend angemessen & 13,73 & 16,61 \\
\hline neutral & 8,14 & 14,07 \\
\hline weitgehend unangemessen & 13,90 & 12,71 \\
\hline ganz unangemessen & 11,86 & 13,39 \\
\hline
\end{tabular}


Bei den Firmennamen Woolworth und Amazon wurden als Varianten die am Englischen orientierten Aussprachen und die eingedeutschten Varianten abgefragt.

\begin{tabular}{|c|c|c|}
\hline & \multicolumn{2}{|c|}{ WOOLWORTH } \\
\hline & ['wu:lws: $\theta$ ] & ['volvort] \\
\hline voll angemessen & 77,70 & 7,60 \\
\hline weitgehend angemessen & 12,25 & 7,60 \\
\hline neutral & 6,13 & 11,27 \\
\hline weitgehend unangemessen & 2,45 & 22,30 \\
\hline ganz unangemessen & 1,47 & 51,23 \\
\hline
\end{tabular}

\begin{tabular}{l|l|l} 
& AMAZON & \multicolumn{2}{|l}{$[(')$ ama(')tso:n $]$} \\
\cline { 2 - 3 } & {$[$ 'Eməzn] $]$} & 51,82 \\
\hline voll angemessen & 46,36 & 20,88 \\
\hline weitgehend angemessen & 20,05 & 12,53 \\
\hline neutral & 13,39 & 8,74 \\
\hline weitgehend unangemessen & 12,54 & 6,03
\end{tabular}

Weiterhin wurde bei Amazon im Falle einer eingedeutschten Aussprache nach der betonten Silbe gefragt.

\begin{tabular}{l|l|l} 
& AMAZON & amaZON \\
\cline { 2 - 3 } & AMazon & 21,50 \\
\hline voll angemessen & 69,89 & 17,71 \\
\hline weitgehend angemessen & 15,17 & 17,94 \\
\hline neutral & 9,29 & 23,20 \\
\hline weitgehend unangemessen & 2,71 & 19,64
\end{tabular}


Auch bei Tupperware ging es um die Wahl zwischen am Englischen orientierter Aussprache und eingedeutschter Variante. Dabei wurden allerdings beide Wortbestandteile getrennt abgefragt. Für das $<\mathrm{u}>$ im Wortbestandteil Tupper- waren die Varianten „der Schreibung entsprechend“, also offenes kurzes $u$ [ $\mho$ ] (wie in Butter), $a$ [a] und offenes kurzes $\ddot{o}$ (wie in Löffel).

\begin{tabular}{l|l|l|l} 
& \multicolumn{2}{l}{ TUPPERWARE } & \multicolumn{2}{l}{} \\
\cline { 2 - 4 } & T[ช]pper(ware) & T[a]pper(ware) & T[œ]pper(ware) \\
\hline voll angemessen & 60,98 & 37,73 & 3,87 \\
\hline weitgehend angemessen & 14,99 & 19,64 & 2,32 \\
\hline neutral & 8,01 & 10,85 & 3,09 \\
\hline weitgehend unangemessen & 6,98 & 12,14 & 11,86 \\
\hline ganz unangemessen & 9,04 & 19,64 & 78,87
\end{tabular}

Bezüglich des Wortbestandteils -ware lag die Wahl ganz allgemein zwischen englischer und deutscher Variante.

\begin{tabular}{|c|c|c|}
\hline & \multicolumn{2}{|c|}{ TUPPERWARE } \\
\hline & (Tupper)[wev] & (Tupper)[va:rə] \\
\hline voll angemessen & 50,13 & 58,14 \\
\hline weitgehend angemessen & 12,92 & 13,70 \\
\hline neutral & 12,40 & 8,27 \\
\hline weitgehend unangemessen & 12,14 & 9,56 \\
\hline ganz unangemessen & 12,40 & 10,34 \\
\hline
\end{tabular}

Bei dem Namen der Automarke Rolls-Royce wurde die Aussprache des $<0>$ in Rolls thematisiert. Die Varianten waren offenes kurzes $o$ [0] (wie in hoffen) und die am Englischen

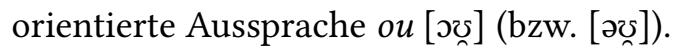

\begin{tabular}{l|l|l} 
& ROLLS-ROYCE & \multicolumn{2}{l}{ R[ơ్ ]lls (-Royce) } \\
\cline { 2 - 3 } & R[o]lls (-Royce) & 25,88 \\
\hline voll angemessen & 62,63 & 13,82 \\
\hline weitgehend angemessen & 19,44 & 19,35 \\
\hline neutral & 9,34 & 20,60 \\
\hline weitgehend unangemessen & 6,57 & 20,35
\end{tabular}


Bei Upgrade wurde auch die Variation bei der Aussprache von $<\mathrm{r}>$ thematisiert. Als Optionen standen die am Englischen orientierte Aussprache (phonetisch ein Approximant bzw. Annäherungslaut, in IPA-Lautschrift $[x]$ ) und die Aussprache wie in deutschen Wörtern (also vor allem Reibe- $r[\mathrm{r}]$ oder regional auch Zungenspitzen-[r]) zur Auswahl.

\begin{tabular}{|c|c|c|}
\hline & \multicolumn{2}{|c|}{ UPGRADE } \\
\hline & Upg[x]ade & Upg[в] ade \\
\hline voll angemessen & 80,15 & 8,44 \\
\hline weitgehend angemessen & 10,67 & 7,44 \\
\hline neutral & 5,46 & 16,87 \\
\hline weitgehend unangemessen & 2,48 & 31,76 \\
\hline ganz unangemessen & 1,24 & 35,48 \\
\hline
\end{tabular}

\section{Schluss}

In den vorhergehenden Abschnitten sind insgesamt zu 207 sprachlichen Variablen Bewertungen zur Angemessenheit von Aussprachevarianten in formellen Sprechsituationen dokumentiert. Von diesen Ergebnissen, die mittels einer im Frühsommer 2015 durchgeführten Online-Umfrage gesammelt wurden, waren bisher nur ausgewählte Teile in der Neuauflage des Duden-Aussprachewörterbuchs (Kleiner/Knöbl/Mangold 2015) publiziert worden. Sie sind mit der hier vorliegenden Publikation erstmals vollständig der Öffentlichkeit zugänglich gemacht worden.

\section{Literatur}

Kleiner, Stefan/Knöbl, Ralf/Mangold, Max (2015): Duden - Das Aussprachewörterbuch. 7., komplett überarbeitete und aktualisierte Auflage. (= Der Duden in 12 Bänden, Bd. 6). Berlin u. a.: Dudenverlag/Mannheim: Institut für Deutsche Sprache.

Kleiner, Stefan (2011ff.): Atlas zur Aussprache des deutschen Gebrauchsstandards (AADG). Unter Mitarbeit von Ralf Knöbl. http://prowiki.ids-mannheim.de/bin/view/AADG/ChemieCh. (Stand: 11.5.2020). 


\section{Bibliografische Informationen}

Angaben zur Zitierung dieser Publikation:

Kleiner, Stefan (2021): Ergebnisse einer Online-Umfrage zur „Angemessenheit von Aussprachevarianten in formellen Sprechsituationen" (= IDSopen 2). Mannheim: IDS-Verlag.

DOI https://doi.org/10.21248/idsopen.2.2021.3

\section{Autorendaten}

Stefan Kleiner

Leibniz-Institut für Deutsche Sprache

R 5, 6-10

68161 Mannheim

E-Mail: kleiner@ids-mannheim.de

\section{Impressum}

\section{Bibliografische Information der Deutschen Nationalbibliothek}

Die Deutsche Nationalbibliothek verzeichnet diese Publikation in der Deutschen

Nationalbibliografie; detaillierte bibliografische Daten sind im Internet über

http://dnb.dnb.de abrufbar.

IDS-Verlag · Leibniz-Institut für Deutsche Sprache

R 5, 6-13 · 68161 Mannheim

www.ids-mannheim.de

\section{IDS IDS-Verlag}

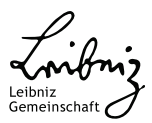

Schriftenreihe: IDSopen: Online-only Publikationen des Leibniz-Instituts für Deutsche Sprache Reihenherausgeber/-innen: Norman Fiedler, Katrin Hein, Silke Reineke, Beata Trawiński Redaktion: Melanie Kraus

Satz: Joachim Hohwieler, Annett Patzschewitz

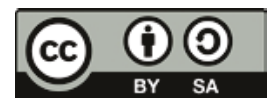

Dieses Werk ist unter der Creative-Commons-Lizenz 3.0 (CC BY-SA 3.0) veröffentlicht.

(2) Diese Publikation erscheint in Open Access. Sie ist auf den Webseiten der IDSopenDie gesetzliche Verpflichtung über die Ablieferung digitaler Publikationen als Pflichtexemplare wird durch die Aufnahme der Netzpublikation in die Datenbank des Bibliotheksservice-Zentrums Baden-Württemberg (BSZ) erfüllt.

ISBN: 978-3-937241-81-4 (PDF)

ISSN: 2749-9855

(c) 2021 Stefan Kleiner 